\title{
The effect of ram pressure on the star formation, mass distribution and morphology of galaxies
}

\author{
W. Kapferer ${ }^{1}$, C. Sluka ${ }^{1}$, S. Schindler ${ }^{1}$, C. Ferrari ${ }^{1,2}$, and B. Ziegler ${ }^{3}$ \\ 1 Institut für Astro- und Teilchenphysik, Universität Innsbruck, Technikerstrasse 25, 6020 Innsbruck, Austria \\ e-mail: wolfgang.e.kapferer@uibk.ac.at \\ 2 Laboratoire Cassiopée, CNRS, UMR 6202, Observatoire de la Côte d'Azur, BP 4229, 06304 Nice Cedex 4, France \\ 3 European Southern Observatory, Karl-Schwarzschild-Strasse 2, 85748 Garching, Germany
}

Received 19 December 2008 / Accepted 14 March 2009

\begin{abstract}
Aims. We investigate the dependence of star formation and the distribution of the components of galaxies on the strength of ram pressure. Several mock observations in X-ray, $\mathrm{H} \alpha$ and $\mathrm{HI}$ wavelength for different ram-pressure scenarios are presented.

Methods. By applying a combined $N$-body/hydrodynamic description (GADGET-2) with radiative cooling and a recipe for star formation and stellar feedback 12 different ram-pressure stripping scenarios for disc galaxies were calculated. Special emphasis was put on the gas within the disc and in the surroundings. All gas particles within the computational domain having the same mass resolution. The relative velocity was varied from $100 \mathrm{~km} \mathrm{~s}^{-1}$ to $1000 \mathrm{~km} \mathrm{~s}^{-1}$ in different surrounding gas densities in the range from $1 \times 10^{-28}$ to $5 \times 10^{-27} \mathrm{~g} / \mathrm{cm}^{3}$. The temperature of the surrounding gas was initially $1 \times 10^{7} \mathrm{~K}$.

Results. The star formation of a galaxy is enhanced by more than a magnitude in the simulation with a high ram-pressure $(5 \times$ $10^{-11} \mathrm{dyn} / \mathrm{cm}^{2}$ ) in comparison to the same system evolving in isolation. The enhancement of the star formation depends more on the surrounding gas density than on the relative velocity. Up to $95 \%$ of all newly formed stars can be found in the wake of the galaxy out to distances of more than $350 \mathrm{kpc}$ behind the stellar disc. Continuously stars fall back to the old stellar disc, building up a bulge-like structure. Young stars can be found throughout the stripped wake with surface densities locally comparable to values in the inner stellar disc. Ram-pressure stripping can shift the location of star formation from the disc into the wake on very short timescales. As the gas in a galaxy has a complex velocity pattern due to the rotation and spiral arms, the superposition of the internal velocity field and the ram pressure causes complex structures in the gaseous wake which survive dynamically up to several 100 Myr. Finally we provide simulated X-ray, $\mathrm{H} \alpha$ and $\mathrm{HI}$ observations to be able to compare our results with observations in these wavebands. These simulated observations show many features which depend strongly both on the strength and the duration of the external ram pressure.
\end{abstract}

Key words. galaxies: clusters: general - galaxies: abundances - galaxies: interactions - galaxies: ISM - X-ray: galaxies: clusters

\section{Introduction}

Since the late 1970s observations have revealed an evolution of galaxy cluster members with redshift. It has been found that the fraction of star-forming and post-star-forming systems increases significantly with redshift (Dressler et al. 1987, 1999). In addition Butcher \& Oemler (1978) showed that there is a strong evolution from bluer to redder colours in cluster galaxies. They have found an excess of blue objects at $z=0.5$ with respect to lower redshift systems, the so-called Butcher-Oemler effect. Many different mechanisms have been studied with special emphasis on their effect on the morphology and the star formation of cluster galaxies. Among these mechanisms ram-pressure stripping (e.g. Gunn \& Gott 1972), galaxy harassment (e.g. Moore et al. 1998) or strangulation/starvation (e.g. Larson et al. 1980) can be found. How strong these different processes influence the evolution of cluster galaxies is still under debate. It is crucial to identify and disentangle different interaction processes, also at intermediate and high redshift. One possible way is to study the evolution of the star formation activity in galaxies and the dependence of this quantity on the galaxies environment (Balogh et al. 2002; Gerken et al. 2004; Poggianti et al. 2006; Verdugo et al. 2008). As the physical processes involved in star formation are poorly understood, additional properties of galaxies were investigated to study whether a galaxy has an interaction with its environment or not. The total gravitational potential of galaxies and its distortions due to interactions with their direct surroundings can be used to disentangle between external and internal processes of galaxy evolution. Technically it is nowadays possible to observe the full 2D velocity field (VF) of local galaxies in optical wavebands using integral field units (IFUs), i.e. SAURON (e.g. Ganda et al. 2006) or Fabry-Perot interferometry (e.g. Chemin et al. 2006; Garrido et al. 2002).

Another approach are numerical simulations to study individually the effects of the various processes. Kronberger et al. (2006, 2007) investigated in numerical simulations the effects of galaxy-galaxy mergers and tidal interactions between galaxies on the internal kinematics of galaxies. The results of these simulations were also used to identify observational biases in observations of the velocity field of distant galaxies (Kapferer et al. 2006; Kronberger et al. 2007). Although dependencies on the viewing angle and on the spatial resolution have been found, it was shown that tidal interactions mainly introduce nonaxisymmetric and nonbisymmetric features (see also Rubin et al. 1999).

From combined X-ray and optical observations of galaxy clusters it was found that the so called intra-cluster medium (ICM), a hot $\left(10^{7}<T<10^{8} \mathrm{~K}\right)$ thin $\left(\rho \sim 1 \times 10^{-27} \mathrm{~g} / \mathrm{cm}^{3}\right)$ plasma, harbours five times more mass than the galaxies themselves. This plasma exerts a pressure on the inter-stellar medium (ISM) of the cluster galaxies, which removes gas from the 
Table 1. Initial properties of the model galaxy.

\begin{tabular}{c|ccc}
\hline \hline & $\begin{array}{c}\text { Number of } \\
\text { particles }\end{array}$ & $\begin{array}{c}\text { Mass resolution } \\
{\left[M_{\odot} / \text { particle }\right]}\end{array}$ & $\begin{array}{c}\text { Total mass } \\
{\left[M_{\odot}\right]}\end{array}$ \\
\hline DM halo & $3 \times 10^{5}$ & $3.5 \times 10^{6}$ & $1.05 \times 10^{12}$ \\
gaseous disc & $2 \times 10^{5}$ & $3.4 \times 10^{4}$ & $6.80 \times 10^{9}$ \\
stellar disc & $2 \times 10^{5}$ & $1.0 \times 10^{5}$ & $2.00 \times 10^{10}$ \\
\hline
\end{tabular}

disc if the force due to the external ram pressure exceeds the restoring gravitational force of the galaxy. The effect of rampressure stripping on individual galaxies was investigated by several groups. The dependence of the stripping radius on the external ram pressure and the galaxy properties was investigated (e.g. Abadi et al. 1999; Vollmer et al. 2001; Roediger $\&$ Hensler 2005) and a remarkable agreement between analytical estimates and numerical simulations was found. Further galaxy-scale numerical simulations for ram-pressure stripping were presented by Quilis et al. (2000), Mori \& Burkert (2000), Toniazzo \& Schindler (2001), Schulz \& Struck (2001), and Roediger \& Brüggen (2006, 2007). Jachym et al. (2007) used an $N$-body/SPH code to investigate the influence of a time varying ram pressure on spiral galaxies. A closer investigation of the stripped gaseous wake with Eulerian grid techniques were carried out by Roediger \& Brüggen (2008). Recently also the effects of the external ram pressure on the star formation rate of the stripped galaxy were studied (Kapferer et al. 2008; Kronberger et al. 2008). The influence of the stripped gas from the galaxies affected by ram pressure on the metallicity of the ICM was investigated by Schindler et al. (2005), Domainko et al. (2006) and Kapferer et al. (2007a,b) in large scale combined $N$-body/hydrodyanmic simulations.

In this work we investigate the dependence of the star formation in a galaxy on different strengths of ram pressure. We study the influence of different strengths of ram pressure on the distribution of the components of a galaxy, i.e. the resulting stellar and gas distribution. In addition we provide mock observations in the radio, the optical and the $\mathrm{X}$-ray range.

\section{The simulation setup}

\subsection{The hydrodynamic description}

The simulations were carried out with the $N$-body/SPH code GADGET-2 developed by Springel (see Springel 2005, for details). The code treats the gas of the galaxies and the surrounding gas by smoothed particle hydrodynamics (SPH Gingold \& Monaghan 1977; Lucy 1977). The collisionless dynamics of the dark matter and the stellar component is modelled by an $N$-body technique. Additional routines for cooling, star formation (SF), stellar feedback, and galactic winds are included as described in Springel \& Hernquist (2003).

\subsection{The initial model for the model galaxies}

The model galaxies were created with an initial disc galaxy generator developed by Volker Springel. Details and analysis can be found in Springel et al. (2004). The model galaxy has a stellar disc-scale length of $3.3 \mathrm{kpc}$ and a halo circular velocity of $160 \mathrm{~km} \mathrm{~s}^{-1}$. The initial gas fraction in the disc is $25 \%$ of the total disc mass. The mass resolution of the different components of the galaxies (gas, stellar, dark matter) is listed in Table 1. In Fig. 1 the gas and stellar distribution of the model galaxy is shown after 2 Gyr of evolution without external ram pressure.

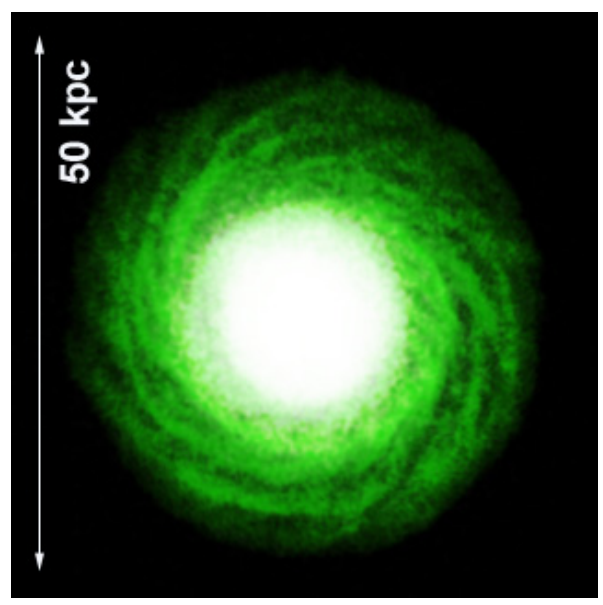

Fig. 1. The distribution of gaseous (green) and stellar (white) mass of the model galaxy after 2 Gyr of isolated evolution.

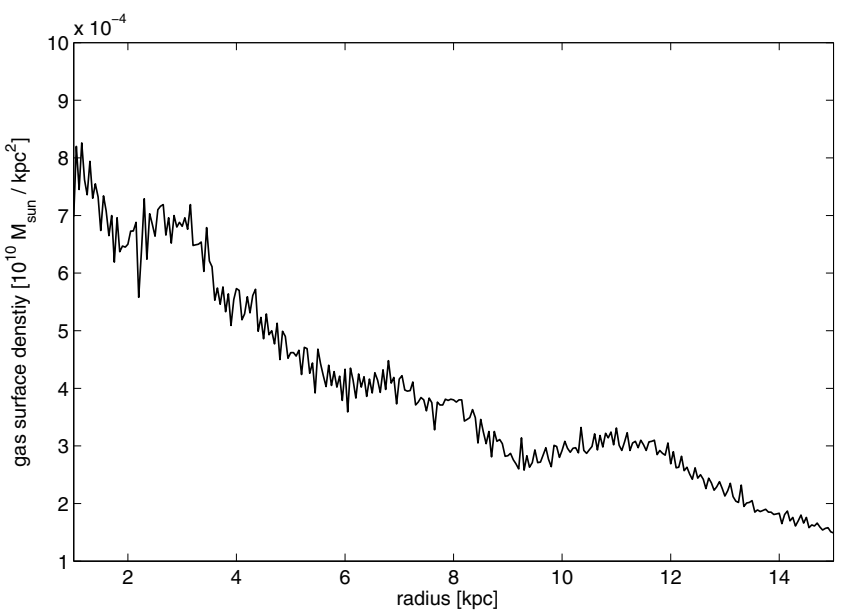

Fig. 2. The radial gas profile of the model galaxy after $2 \mathrm{Gyr}$ of isolated evolution.

After $2 \mathrm{Gyr}$ of isolated evolution, i.e. no surrounding gas, $2.2 \times 10^{10} M_{\odot}$ new stars have formed and the actual star formation rate is $1.6 M_{\odot} / y r$. In Fig. 2 the density profile of the gas for the model galaxy after 2 Gyr of evolution is shown. The several local maxima originate from the pattern of the gaseous spiral arms.

\subsection{The star formation and feedback model}

We applied, as in Kronberger et al. (2008) and Kapferer et al. (2008), the so called hybrid method for star formation and stellar feedback, introduced by Springel \& Hernquist (2003). The fundamental assumption of this model is the conversion of cold clouds into stars on a characteristic timescale $t_{*}$ and the release of a certain mass fraction $\beta$ due to supernovae ( $\mathrm{SNe}$ ). The minimum temperature the gas can reach due to radiative cooling is $10^{4} \mathrm{~K}$. From observations and analytical models it was found, that matter can escape the galaxies potential due to thermal and/or cosmic-ray driven winds caused by SN explosions (Breitschwerdt et al. 1991). We applied the same method as Springel \& Hernquist (2003) and selected the mass of the outflow such, that the mass of the galactic wind is direct proportional to the actual star formation rate with a proportionally factor of two. More details on the model can be found in Springel \& Hernquist (2003). 


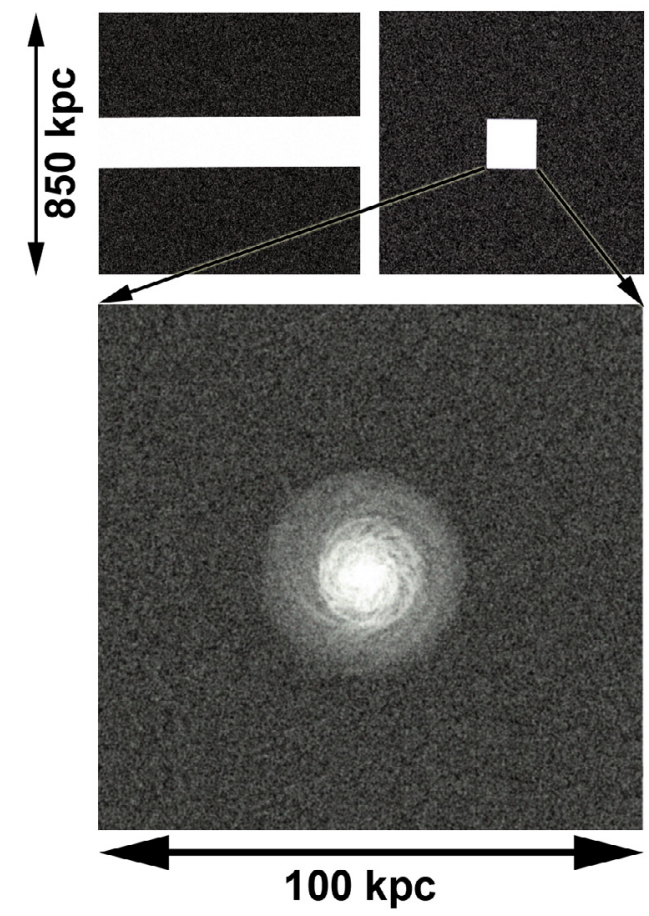

Fig. 3. The simulation box with the high resolution gaseous wind tunnel in the middle, surrounded by a low resolution region. In the upper panels the computational domain is shown from the side and from the top, whereas in the lower panel a the inner (highly resolved) wind tunnel with the galaxy is seen. The greyscale reflects the particle density.

\subsection{The ram pressure}

To study the dependence of the star-formation rate and the morphology of a galaxy on the strength of an external ram pressure, we expose the model galaxy after $2 \mathrm{Gyr}$ of isolated evolution to an external wind. The galaxy moves then with a constant velocity through the ambient medium in a face-on orientation. The simulations are set up in the same way as in Kronberger et al. (2008) and Kapferer et al. (2008) with a modification regarding the mass resolution of the gas particles. The simulation box has $850 \mathrm{kpc}$ on a side and periodic boundaries. In order to obtain the same mass resolution for the gas in the model galaxy and in the surrounding gas a high resolution wind tunnel is set in the simulation box, which is surrounded by a less resolved medium, in order to keep the wind tunnel's hydrodynamic properties stable during the simulation time which is 1 Gyr. In Fig. 3 the simulation box is shown. In Fig. 4 the gas particle distribution of the surrounding gas at the beginning and after $1 \mathrm{Gyr}$ of evolution for simulation 1 (see Table 2) is shown. The mean density of the wind tunnel does not change more than $4 \%$ over 1 Gyr of simulation time for all chosen density configurations, which leads to a nearly constant ram pressure on the model galaxy during the whole simulation.

Table 2 gives the details on the strength of the ram pressure for all simulations. In total, twelve different strengths of ram pressure acting face-on on the model galaxy are simulated. The values for the surrounding gas densities and the relative velocities represent a wide range of ram pressure acting on galaxies in galaxy clusters. We know from X-ray observations of galaxy clusters, that the density from the ICM is in the range of several $10^{-26} \mathrm{~g} / \mathrm{cm}^{-3}$ (i.e. several $10^{-2} \mathrm{~cm}^{-3}$ in number density) in the central regions down to several $10^{-28} \mathrm{~g} / \mathrm{cm}^{-3}$ (i.e. several $10^{-4} \mathrm{~cm}^{-3}$ in number density) in the outskirts

\section{initial conditions for wind tunnel}
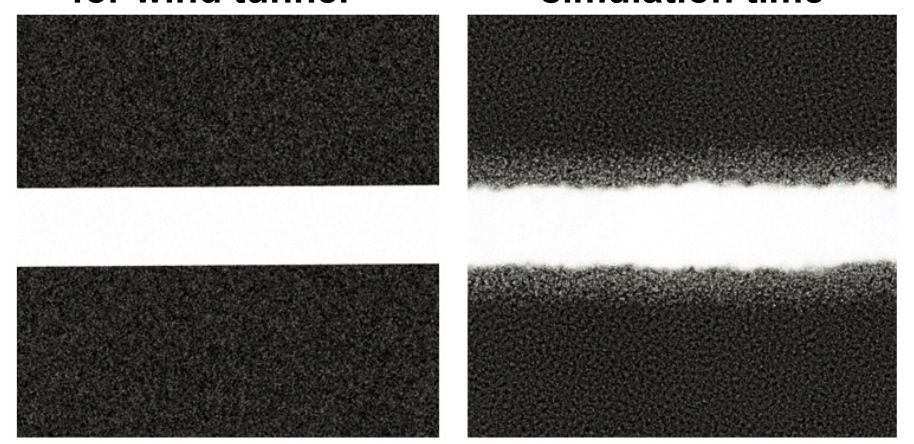

Fig. 4. The simulation box with the high resolution gaseous wind tunnel in the middle shown at the start of the simulation and after $1 \mathrm{Gyr}$ of evolution. The greyscale reflects the particle density.

Table 2. The properties of the ram pressure and the surrounding gas of the simulations.

\begin{tabular}{c|cccc}
\hline \hline run & $\begin{array}{c}v_{\text {rel }} \\
{\left[\mathrm{km} \mathrm{s}^{-1}\right]}\end{array}$ & $\begin{array}{c}\rho_{\text {sur }} \\
{\left[\mathrm{g} / \mathrm{cm}^{3}\right]}\end{array}$ & $\begin{array}{c}\text { particle } \\
\text { number }\end{array}$ & $\begin{array}{c}p \\
{\left[\mathrm{dyn} \mathrm{cm}^{-2}\right]}\end{array}$ \\
\hline 1 & 1000 & $1 \times 10^{-28}$ & $1.08 \times 10^{6}$ & $1.00 \times 10^{-12}$ \\
2 & 1000 & $5 \times 10^{-28}$ & $5.40 \times 10^{6}$ & $5.00 \times 10^{-12}$ \\
3 & 1000 & $1 \times 10^{-27}$ & $1.08 \times 10^{7}$ & $1.00 \times 10^{-11}$ \\
4 & 1000 & $5 \times 10^{-27}$ & $5.40 \times 10^{7}$ & $5.00 \times 10^{-11}$ \\
5 & 500 & $1 \times 10^{-28}$ & $1.08 \times 10^{6}$ & $2.50 \times 10^{-13}$ \\
6 & 500 & $5 \times 10^{-28}$ & $5.40 \times 10^{6}$ & $1.25 \times 10^{-12}$ \\
7 & 500 & $1 \times 10^{-27}$ & $1.08 \times 10^{7}$ & $2.50 \times 10^{-12}$ \\
8 & 500 & $5 \times 10^{-27}$ & $5.40 \times 10^{7}$ & $1.25 \times 10^{-11}$ \\
9 & 100 & $1 \times 10^{-28}$ & $1.08 \times 10^{6}$ & $1.00 \times 10^{-14}$ \\
10 & 100 & $5 \times 10^{-28}$ & $5.40 \times 10^{6}$ & $5.00 \times 10^{-14}$ \\
11 & 100 & $1 \times 10^{-27}$ & $1.08 \times 10^{7}$ & $1.00 \times 10^{-13}$ \\
12 & 100 & $5 \times 10^{-27}$ & $5.40 \times 10^{7}$ & $5.00 \times 10^{-13}$ \\
\hline
\end{tabular}

$v_{\text {rel }}$ : relative velocity between the surrounding gas and the galaxy; $\rho_{\text {sur }}$ : surrounding gas density; $p$ : ram pressure on the galaxy.

(Schindler et al. 1999; Russel et al. 2008), depending on the total mass of the galaxy cluster. From optical spectroscopical investigations it is evident that galaxies have velocities of up to several $1000 \mathrm{~km} \mathrm{~s}^{-1}$ in the centre of galaxy clusters, depending on the dynamical state of the galaxy cluster and the total mass of the system, e.g. Maurogordato et al. (2008). We split our simulation into three major groups, first the fast moving galaxies with a relative velocity of $1000 \mathrm{~km} \mathrm{~s}^{-1}$, second an intermediate relative velocity of $500 \mathrm{~km} \mathrm{~s}^{-1}$ and third a group of very slow moving galaxies with a relative velocity of $100 \mathrm{~km} \mathrm{~s}^{-1}$ with respect to the ambient medium. Note that the temperature of the ambient medium is fixed to $1 \times 10^{7} \mathrm{~K}$. How turbulence, especially Kelvin-Helmholtz instabilities, would affect the gaseous structures in the wake of the stripped galaxies is discussed in Kapferer et al. (2008). Concluding from this previous investigation Kelvin-Helmholtz instabilities affect dense, cool gas knots in the wake, but the Kelvin-Helmholtz timescales for the given densities and the relative velocities are much larger than the dynamical timescales of ram-pressure stripping. This leads to the conclusion that the structures in the gaseous wake can at least survive within the simulation time, which is typically $750 \mathrm{Myr}$. 


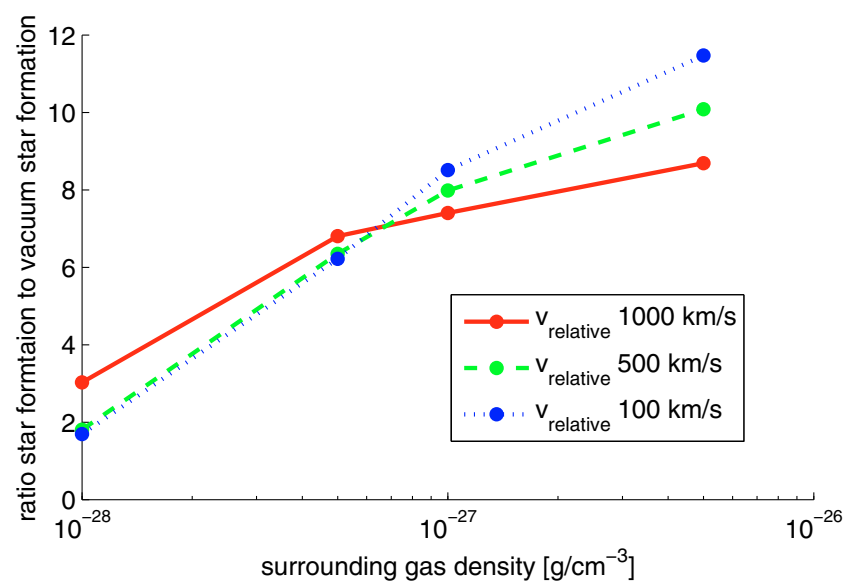

Fig. 5. Ratio of the star formation of the different simulations to the star formation integrated over $500 \mathrm{Myr}$ for the isolated evolving galaxy.

\section{Results}

In this section we investigate the influence of different strengths of ram pressure on the gaseous and stellar components of the model galaxy. In addition we present mock observations of the simulations in different wavelengths regimes. The virtual observations span from radio to optical and X-ray wavelengths.

\subsection{The influence of ram pressure on the star formation}

Recent numerical studies (Kronberger et al. 2008) showed that ram pressure does enhance the star formation in a galaxy. In order to study a relation between the star formation enhancement and the external ram pressure, we calculated the ratio between the temporal integrated-star formation rate of each simulation including an external ram pressure to the case of the isolated galaxy (i.e. no external ram-pressure).

We vary the relative velocities and surrounding gas densities, as summarised in Table 2. The higher the external density, the stronger the star formation enhancement (see Fig. 5). The effect of the relative velocity between the galaxy and the surrounding medium is relatively small. The rate of star-formation enhancement changes depending on the ambient pressure, which depends on the gas density. The rate does nearly not change in the simulations with $v_{\text {rel }}=100 \mathrm{~km} \mathrm{~s}^{-1}$. The reason is the depletion of the gas disc, which can be stripped completely in the case of simulation 4, and the resulting suppression of star formation in the disc. The faster the relative velocity, the larger is the amount of stripped ISM, which will be kinematically stronger disturbed, i.e. kinetic energy will be partly converted in internal - thermal - energy, as in the case with lower relative velocity. This leads to fewer star forming regions than in cases with lower relative velocities.

From observations (e.g. Mihos et al. 2005) it is evident that an intra-cluster stellar population exists. Some observations claim that $10-40 \%$ of all stars in galaxy clusters are in the space between the galaxies (e.g. Feldmeier et al. 1998; Theuns \& Warren 1997; Arnaboldi et al. 2003). From recent numerical studies (Kapferer et al. 2008) we know that ram pressure can cause star formation in the stripped wake of galaxies Therefore we investigated the amount and location of newly formed stars for the different ram-pressure scenarios.

We found a relation between the relative velocity and the amount of star forming regions in the wake. The higher the relative velocity between the galaxy and the surrounding medium,

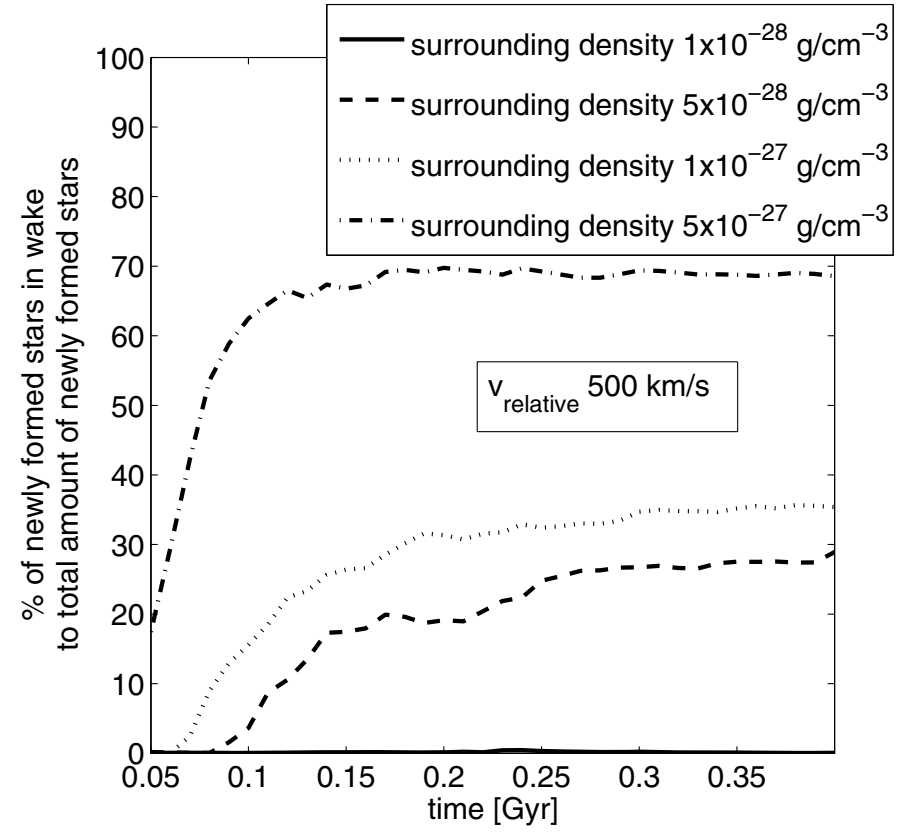

Fig. 6. The evolution of the mass fraction of newly formed stars in the wake (distances larger than $10 \mathrm{kpc}$ above the disc) in comparison to the total amount of newly formed stars for $v_{\text {rel }}=500 \mathrm{~km} \mathrm{~s}^{-1}$. Note that the solid line is, corresponding to the surrounding gas density of $1 \times$ $10^{-28} \mathrm{~g} / \mathrm{cm}^{-3}$, is almost constantly zero, i.e. no stars are formed at distances larger than $10 \mathrm{kpc}$ behind the disc.

the more star forming region are developing in the wake. The explanation is the fact, that stronger external ram pressure strips off more gas from the disc, which leads to a depletion of gas in the galaxy. The highest ratio, i.e. nearly all new stars being formed in the wake, can be found in the simulation with the highest surrounding gas density and the highest relative velocity.

In the case of the lowest relative velocity (i.e. $100 \mathrm{~km} \mathrm{~s}^{-1}$ ) almost no new stars are formed in the wake, because nearly no gas is stripped in these scenarios. Note that the wake is defined as region above $10 \mathrm{kpc}$ the stellar disc (i.e. the same definition as in Kronberger et al. 2008).

In Figs. 6 and 7 the evolution of the amount of newly formed stars in the wake relative to the total amount of newly formed stars is shown. In the case of $1000 \mathrm{~km} \mathrm{~s}^{-1}$ relative velocity and a surrounding gas density of $5 \times 10^{-27} \mathrm{~g} / \mathrm{cm}^{3}$ more than $95 \%$ of all new stars are formed in the wake, as the disc as stripped almost completely. In the scenarios with a relative velocity of $500 \mathrm{~km} \mathrm{~s}^{-1}$, the amount decreases to nearly $70 \%$ in the case with the highest surrounding gas density. Note that the ratio of star formation in the disc to the wake saturates at different timescales, depending on the strength of the ram pressure. We find timescales in the range from several $50 \mathrm{Myr}$ in the strongest ram pressure cases to nearly $300 \mathrm{Myr}$ in the scenarios with the lowest surrounding gas densities. This behaviour reflects the ram-pressure stripping time scales, i.e. the time until the ram pressure has stripped the gaseous disc to a radius of equilibrium between internal and external forces, i.e. the stripping radius.

As a next step we investigated the amount of newly formed stars in the stripped wake as a function of the ram pressure. In Fig. 8 the total mass of newly formed stars as a function of the surrounding gas density is shown. It is remarkable that nearly $1 \times 10^{9} M_{\odot}$ of stellar mass is present in the wake after 500 Myrs of evolution in the strongest ram pressure scenario. Even in the case of a very low relative velocity of $100 \mathrm{~km} \mathrm{~s}^{-1} 1 \times 10^{8} M_{\odot}$ 


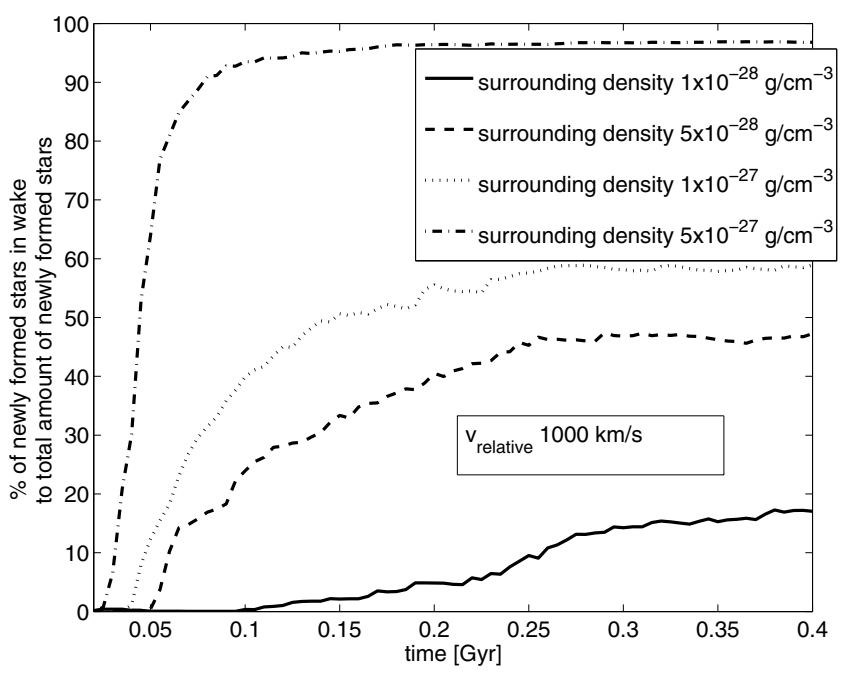

Fig. 7. The evolution of the fraction of newly formed stars in the wake (distances larger than $10 \mathrm{kpc}$ above the disc) in comparison to the total amount of newly formed stars for $v_{\text {rel }}=1000 \mathrm{~km} \mathrm{~s}^{-1}$.

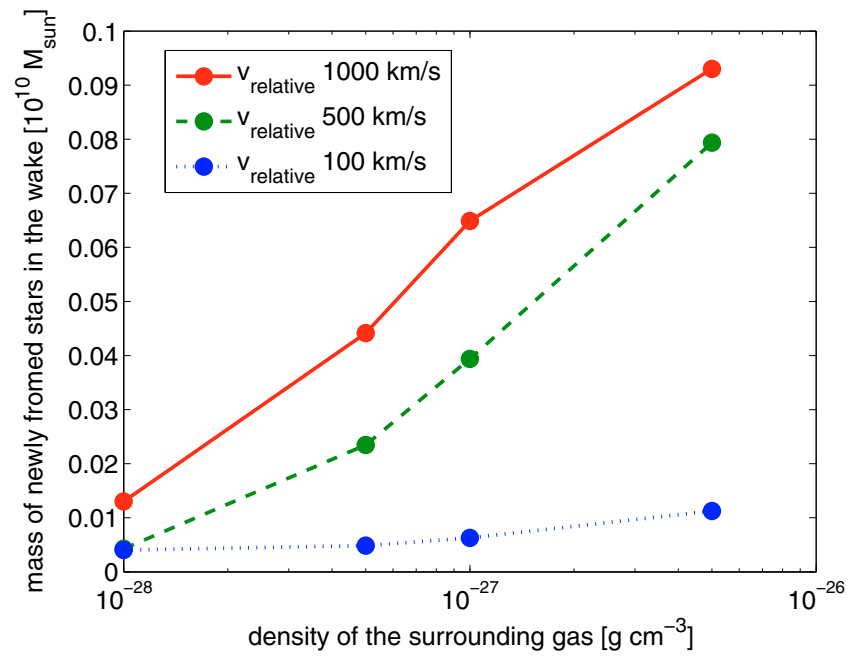

Fig. 8. Mass of newly formed stars in the wake (distances larger than $10 \mathrm{kpc}$ above the disc) after $500 \mathrm{Myr}$ of evolution.

of stellar matter can be found at distances larger than $10 \mathrm{kpc}$ to the disc.

How the morphology of the stellar distribution is affected by different strengths of ram pressure can be seen in Figs. 9 and 12, respectively. The two figures show the stellar distribution after $500 \mathrm{Myr}$ of ram pressure acting on the galaxy. The isocontours show the projected stellar density for the different ram-pressure scenarios. The surface density varies by five orders of magnitudes, with the maximum at the very centre of the disc. The stronger the ram pressure the more gas is stripped from the galaxies (see next section for details) which acts as gas reservoir for new stars. Assuming a constant mass to light ratio these two figures can be directly interpreted as optical observations. In the cases of $v_{\text {rel }}=500 \mathrm{~km} \mathrm{~s}^{-1}$ three times less luminous stellar regions than in the central parts are seen up to $120 \mathrm{kpc}$ behind the disc (see Fig. 9). In the cases with $v_{\text {rel }}=1000 \mathrm{~km} \mathrm{~s}^{-1}$ the distances can reach up to $400 \mathrm{kpc}$. In the case highlighted as (d) in Fig. 12 a stellar "highway" with luminosities four to five orders of magnitudes lower than in the central disc are present.

The distribution of newly formed stars within the last $50 \mathrm{Myr}$ can be seen in Figs. 10 and 11, respectively. The two simulations, i.e. run 1 and 2, are shown here in an exemplary way. The chosen time interval of $50 \mathrm{Myr}$ is the approximate life time of less massive B stars in OB-associations. These associations would be observable in $\mathrm{H} \alpha$. In Fig. 10 the distribution of all stars (grey scale) and the young stars (formed within the last $50 \mathrm{Myr}$; isolines) are shown in a logarithmic scale. The timesteps (a)-(d) correspond to $50 \mathrm{Myr}, 250 \mathrm{Myr}, 500 \mathrm{Myr}$ and $750 \mathrm{Myr}$ after the ram pressure started to act on the galaxy face on. These young stellar components can be seen throughout the simulation in both components, in the remaining gaseous disc and in the stripped wake. In simulation 2 (Fig. 11) the same trend can be seen. The stronger the ram pressure, the smaller the star forming regions in the remaining disc. In this case more $\mathrm{OB}$-association like regions can be found in the wake. One important conclusion is, that an overall high star formation rate does not imply high $\mathrm{H} \alpha$ fluxes originating from the disc, but more $\mathrm{H} \alpha$ flux observable in the wake. But as the surface brightness drops one would observe less star forming systems due to observational limitations, although more stars are formed compared to the isolated case. Simulations 1 and 2 can be related as low ram-pressure scenarios in comparison to the strength of ram pressure expected in massive galaxy clusters. Nevertheless the relatively low ram pressure changes the morphology of the galaxy on very short timescales (less than $250 \mathrm{Myr}$ ). From an observational point of view the system would change from a star-forming, blue system, to a red, passively evolving system, although stronger star formation is present as in the isolated scenario. Therefore we can conclude here, that ram pressure enhances the overall star formation of disc galaxies and alters their star formation morphology locally, from star forming discs to star forming wakes.

In addition to the stellar components at large distances from the disc in the stripped wake an additional stellar component is present in front of the disc, opposite to the stripped wake. The stars originate from the gaseous wake in which they were originally formed. As the stars do not feel the ram pressure of the surrounding gas after they have formed, gravity is the the only force they are exposed to. Therefore they will free-fall to the gravitational centre, which is the disc embedded in the dark-matter halo. As the interaction is collisionless, stars will pass through the centre and appear on the other side and oscillate around the centre of the disc leading to an observable component in the opposite direction of the ram pressure. The same behaviour can be observed in the stripped gas, whenever gas is moving into the slipstream of the remaining gas in the disc, it partly falls back onto the disc. More details on the influence of ram pressure on the ISM is given in the next sections.

\subsection{The influence of ram pressure on the gaseous disc}

How different strengths of ram pressures will affect the gaseous disc of the galaxy will be investigated in this section. In Fig. 13 the ratio of the gas mass in the wake to the total gas mass as a function of the surrounding gas density for the three different relative velocities after $500 \mathrm{Myr}$ of evolution is shown. As the ram pressure depends on the product of the square of the relative velocity and the surrounding gas density the increase for the scenarios with $v_{\text {rel }}=1000 \mathrm{~km} \mathrm{~s}^{-1}$ and $v_{\text {rel }}=500 \mathrm{~km} \mathrm{~s}^{-1}$ is stronger than the increase for $v_{\text {rel }}=100 \mathrm{~km} \mathrm{~s}^{-1}$.

Even low gas densities can deplete the gas mass in the disc. Up to $40 \%$ of the gas mass in the disc is reduced in the case of the lowest density of the surrounding gas and a relative velocity of $1000 \mathrm{~km} \mathrm{~s}^{-1}$. If the gas density is larger than $5 \times 10^{-27} \mathrm{~g} / \mathrm{cm}^{3}$ the gas reduction in the disc is strong, the gaseous disc vanishes in the scenario with $1000 \mathrm{~km} \mathrm{~s}^{-1}$ and only $20 \%$ of the gaseous 

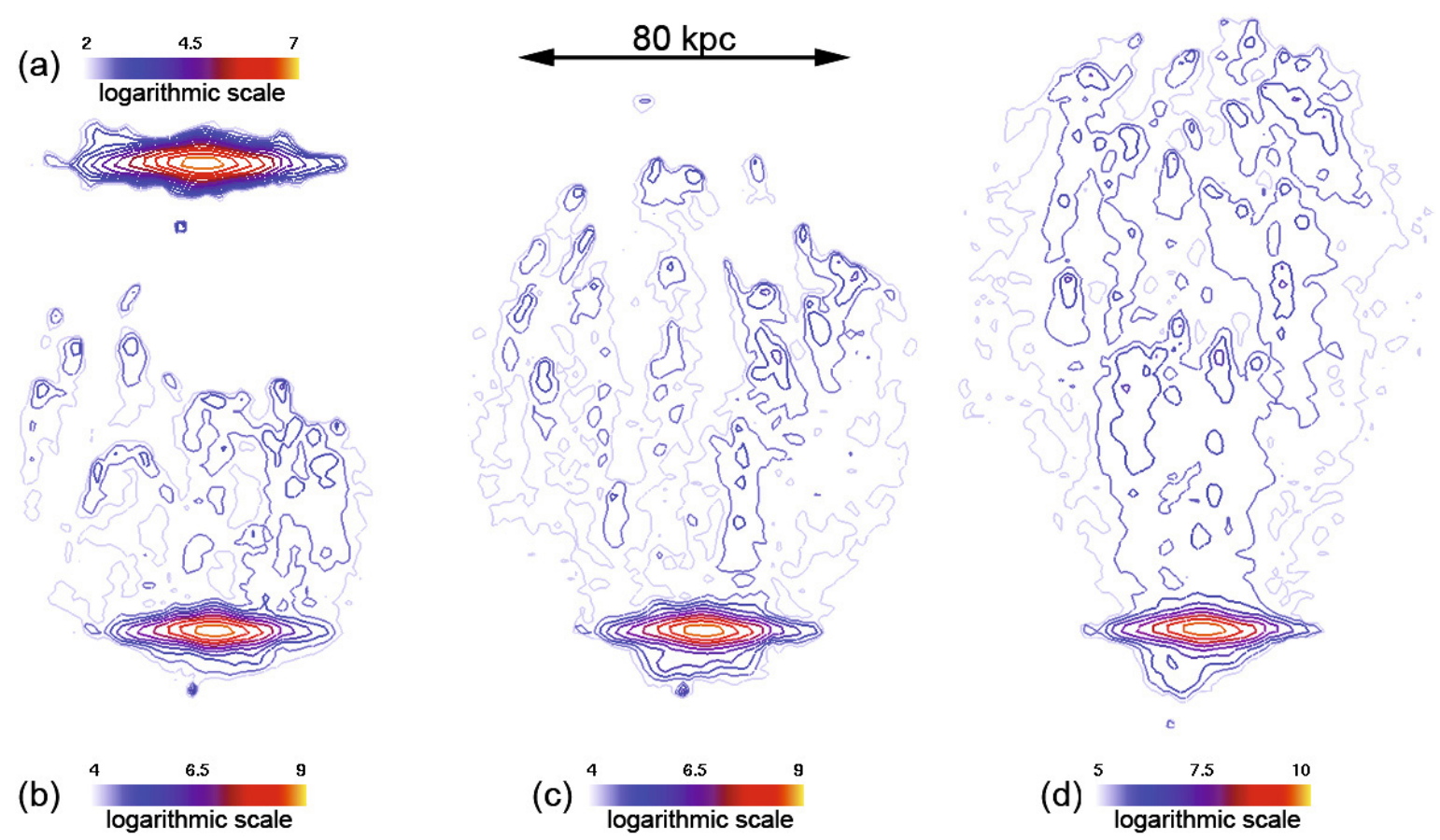

Fig. 9. The surface density of the total stellar component (in relative units and a logarithmic scale) after 500 Myr of ram pressure acting on the galaxy. The relative velocity is $500 \mathrm{~km} \mathrm{~s}^{-1}$ for all shown cases, whereas the surrounding density is varied (a) $\left.1 \times 10^{-28} \mathrm{~g} / \mathrm{cm}^{3}, \mathbf{b}\right) 5 \times 10^{-28} \mathrm{~g} / \mathrm{cm}^{3}$, c) $1 \times 10^{-27} \mathrm{~g} / \mathrm{cm}^{3}$, (d) $5 \times 10^{-27} \mathrm{~g} / \mathrm{cm}^{3}$ ). Note that the panels have different scales (see colour bars).

disc survives in the scenario with $500 \mathrm{~km} \mathrm{~s}^{-1}$. Note that even a very low relative velocity of $100 \mathrm{~km} \mathrm{~s}^{-1}$ does reduce the gas disc by $10 \%$ in the case with $5 \times 10^{-27} \mathrm{~g} / \mathrm{cm}^{3}$ surrounding gas density.

The timescales of gas depletion in the disc are shown in Figs. 14 and 15, respectively. The higher the ram pressure the faster an equilibrium state of ram pressure and restoring, gravitational, forces is established. The fastest gas depletion in the maximum ram pressure scenario $\left(v_{\text {rel }}=1000 \mathrm{~km} \mathrm{~s}^{-1}\right.$ and surrounding gas density of $5 \times 10^{-27} \mathrm{~g} / \mathrm{cm}^{3}$ ) is reached after $100 \mathrm{Myr}$. It is interesting that the equilibrium state is always reached in timescales below 150-200 Myr. Only in the scenario with $500 \mathrm{~km} \mathrm{~s}^{-1}$ relative velocity and the lowest surrounding gas density $1 \times 10^{-28} \mathrm{~g} / \mathrm{cm}^{3}$ does it take $250 \mathrm{Myr}$ to reach the maximum, but as a large amount of gas falls back to the disc the amount of stripped gas is less. Again the effect of a slipstream caused by the the remaining gas disc can be observed. The same effect can be seen in the simulation with $100 \mathrm{~km} \mathrm{~s}^{-1}$ relative velocity and a surrounding gas density of $5 \times 10^{-28} \mathrm{~g} / \mathrm{cm}^{3}$.

\subsection{The distribution of the different components}

In this section it is investigated how ram pressure distributes the gaseous component of the galaxy in the surrounding region. Therefore we calculate for several simulations mock observations in the different wavelengths, i.e. HI and X-ray observations. In Figs. 20 and 21 the projected gas density of all the gas which belongs to the galaxy can be seen. In the upper panel a resolution of $3 \mathrm{kpc}$ is chosen. In the lower panel the same map is smoothed with a Gaussian with $\sigma=4 \mathrm{kpc}$ to mimic a more realistic beam of a radio telescope for a galaxy observed at a distance of the Virgo galaxy cluster. The cold ISM gas (gas temperature $T<$ $20000 \mathrm{~K}$ ), which would correspond to HI emission is shown in the Figs. 22-24. An interesting feature is the increase of gas concentrations in the wake as a function of increasing ram pressure.
Note that the gas density projection is shown with isolines in a logarithmic scale.

The gas knots in the wake are typically 2 to 3 orders of magnitude less dense as the central parts of the gaseous disc. The origin of these knots lies in the original gas distribution of the undisturbed disc, i.e. the spiral arms. In Fig. 16 the evolution of the gaseous spiral arms for one simulation over $200 \mathrm{Myr}$ of ram pressure is shown in detail. As the disc rotates the ram pressure removes the complex gas distribution in the disc. As the rotating spiral arms are removed from the galaxy, selfgravitating structures of gas form from the spiral arms, which evolve into the star-forming regions in the wake. In Fig. 17 the gas density of the disc in run 1 (external ram pressure is $1 \times 10^{-12} \mathrm{dyn} / \mathrm{cm}^{2}$ ) is shown for 8 different timesteps. A very similar behaviour as reported by Schultz \& Struck (2001) can be seen, the compression of the residual disc and the formation of flocculent spirals. The result is an increase of the star formation in the residual gaseous disc. If the ram pressure is increased the stripping radius moves inwards the disc. This leads to the stripping of denser spiral arms, which stay bound together longer and are less affected by the ram pressure as the outer HI disc. In Fig. 18 the evolution of the mean local density of the gas at the position of the cold $\left(T<10^{6} \mathrm{~K}\right)$ gas, i.e. star forming regions, in the wake for simulations 1 to 4 is shown. The rather constant mean local density in the wake found in all simulations, shows that the stellar feedback process does not destroy the gaseous knots in the wake. They stay bound on very long timescales, more than several hundreds of Myr. In Fig. 19 the integrated volume of all gas structures in the wake with a isosurface density of $1 \times 10^{5} M_{\odot} / \mathrm{kpc}^{3}$ after $500 \mathrm{Myr}$ of ram pressure is shown. As the ram pressure increases the volume, i.e. the number of gas complexes, increases. If the surrounding gas density is increased by a factor of 2 the number of complexes is increased by a factor of 1.6 in the case of $v_{\text {rel }}=1000 \mathrm{~km} \mathrm{~s}^{-1}$. In scenarios with $1000 \mathrm{~km} \mathrm{~s}^{-1}$ the ram pressure shows strong influence on the gas disc and the resulting gas wake includes many complex features. In the case (d) in 

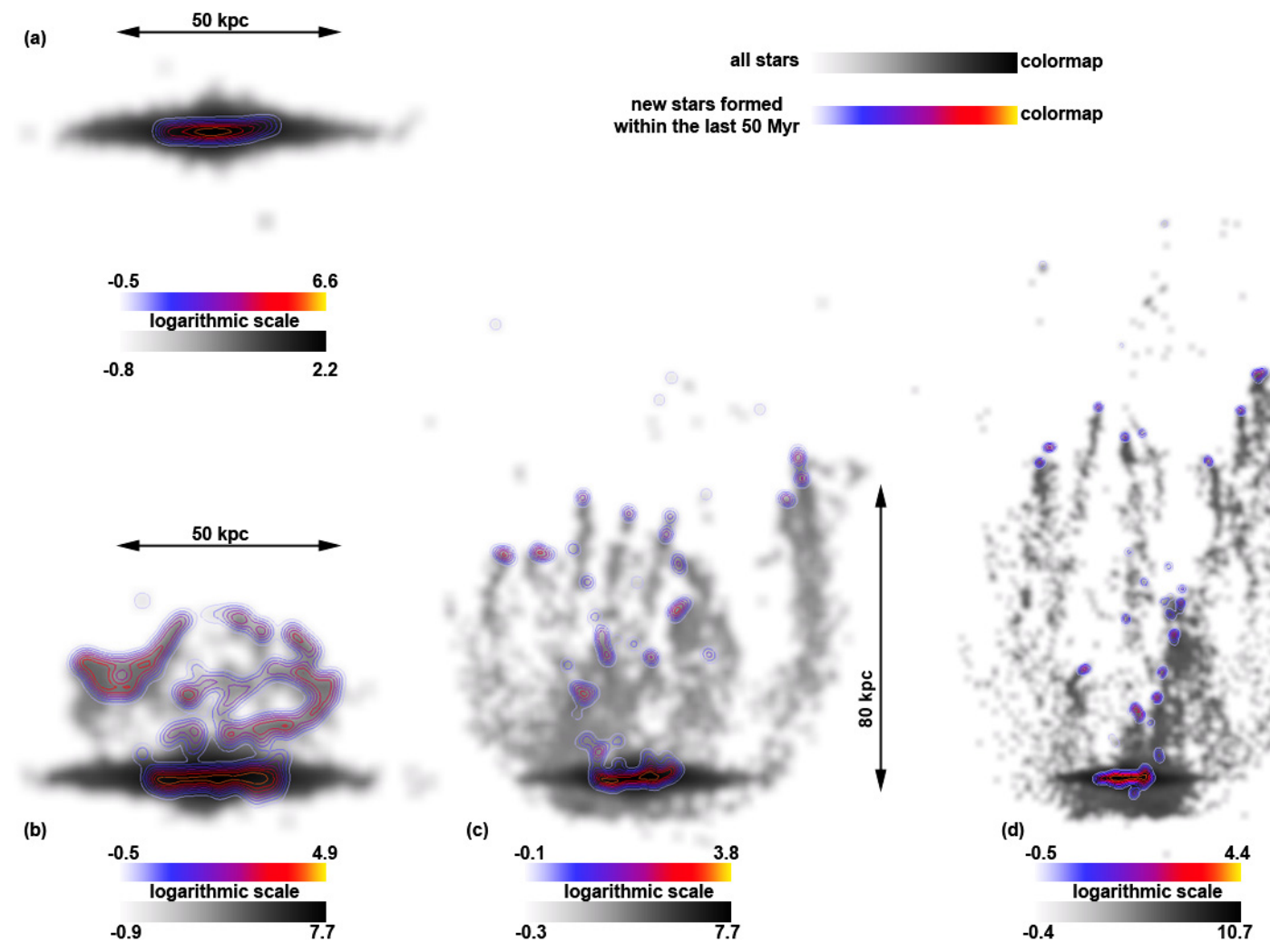

Fig. 10. The surface density of the total stellar component (in grey scale) and newly formed stars within the last 50 Myr (isolines) for different timesteps (a) $50 \mathrm{Myr}$, b) $250 \mathrm{Myr}$, c) $500 \mathrm{Myr}$ and c) 750) of run 1 . The relative velocity is $1000 \mathrm{~km} \mathrm{~s}^{-1}$ and the surrounding gas density is $1 \times$ $10^{-28} \mathrm{~g} / \mathrm{cm}^{3}$. Note that the panels have different scales in the density and length scales.

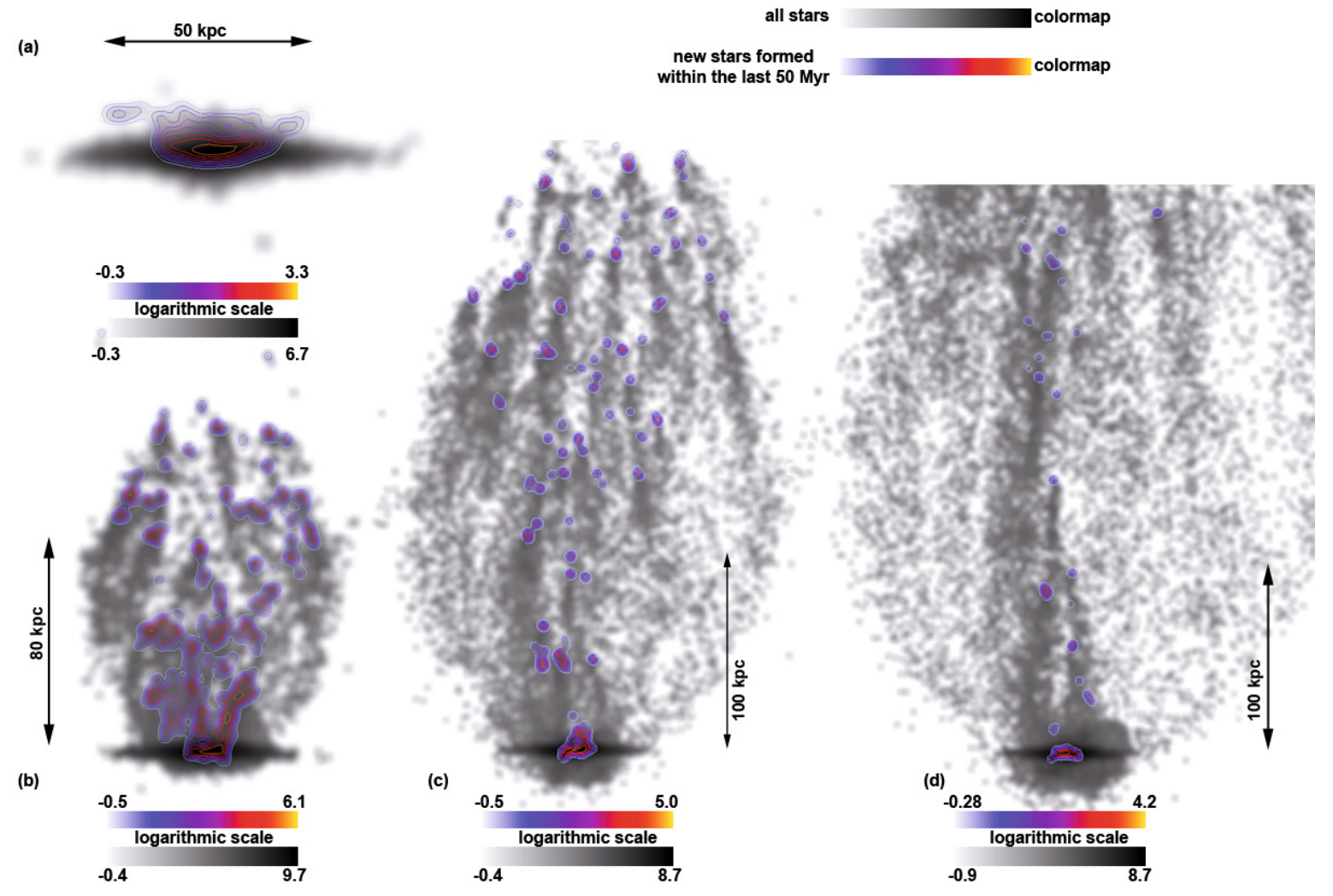

Fig. 11. The surface density of the total stellar component (in grey scale) and newly formed stars within the last 50 Myr (isolines) for different timesteps (a) $50 \mathrm{Myr}$, b) $250 \mathrm{Myr}$, c) $500 \mathrm{Myr}$ and c) 750) in simulation 2 . The relative velocity is $1000 \mathrm{~km} \mathrm{~s}^{-1}$ and the surrounding density is $5 \times$ $10^{-28} \mathrm{~g} / \mathrm{cm}^{3}$. Note that the panels have different scales in the density and length scales. 

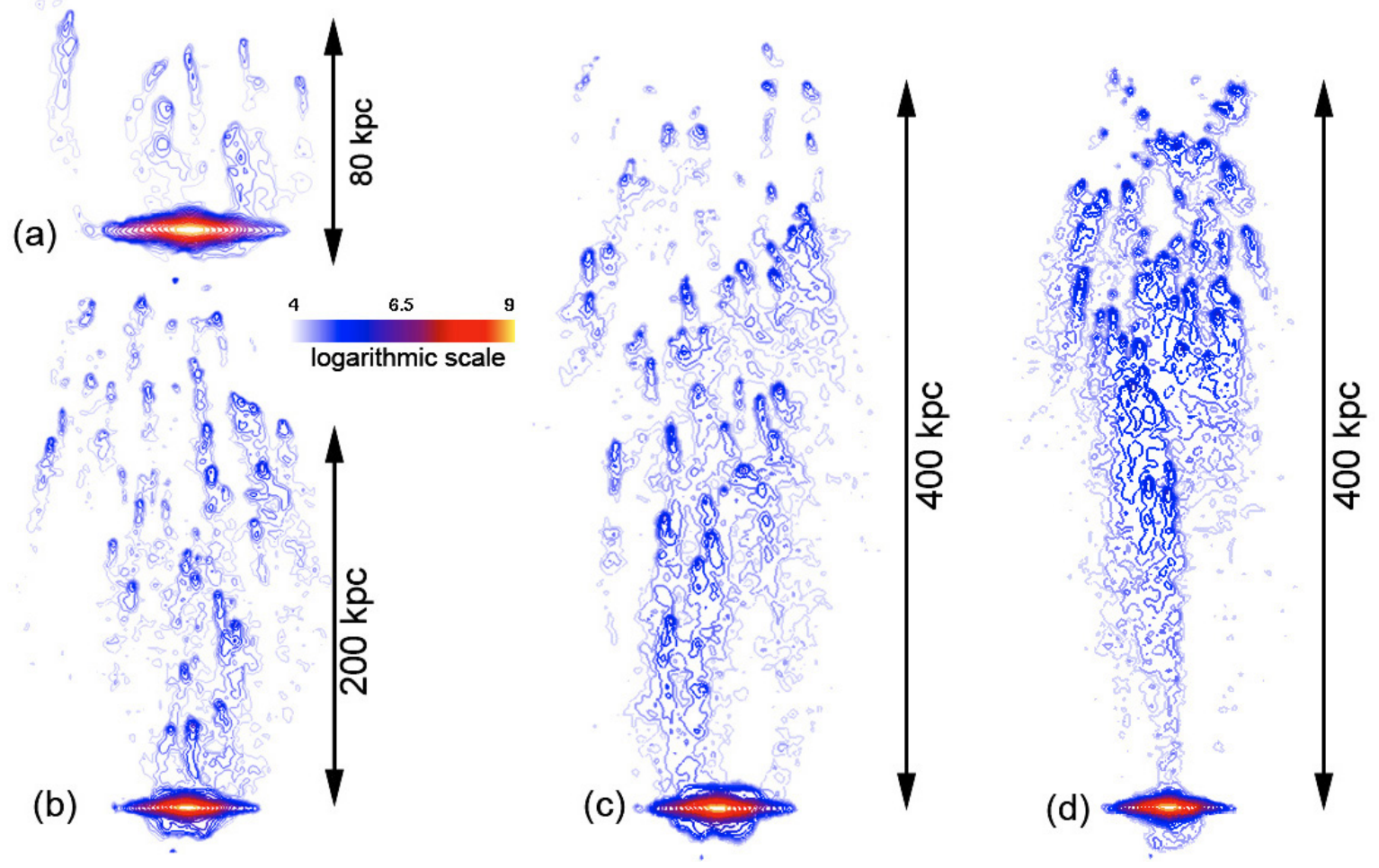

Fig. 12. The surface density of the total stellar component (in relative units and a logarithmic scale) after 500 Myr of ram pressure acting face on. The relative velocity is for all shown cases $1000 \mathrm{~km} \mathrm{~s}^{-1}$, whereas the surrounding density is changed (a) $\left.1 \times 10^{-28} \mathrm{~g} / \mathrm{cm}^{3}, \mathbf{b}\right) 5 \times 10^{-28} \mathrm{~g} / \mathrm{cm}^{3}$, c) $1 \times 10^{-27} \mathrm{~g} / \mathrm{cm}^{3}$, d) $\left.5 \times 10^{-27} \mathrm{~g} / \mathrm{cm}^{3}\right)$.

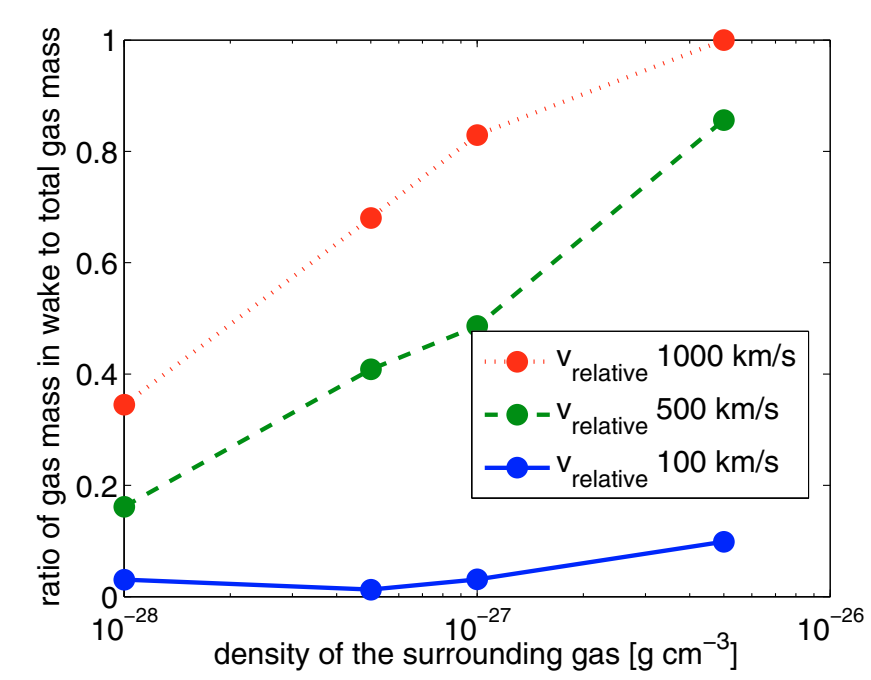

Fig. 13. Ratio of gas mass in the wake to the total gas mass of the system (distances larger than $10 \mathrm{kpc}$ behind the disc) after $500 \mathrm{Myr}$ of evolution for simulations with different densities and $v_{\text {rel }}$.

Fig. 20 the ram pressure has stripped off the whole gaseous disc. The stripped gas lies more than $150 \mathrm{kpc}$ behind the disc after $500 \mathrm{Myr}$ of ram pressure, leading to an elongated gas structure with several gaseous clumps. In the scenarios with with $v_{\text {rel }}=$ $500 \mathrm{~km} \mathrm{~s}^{-1}$ of relative velocities the gaseous disc is not completely stripped. Nevertheless the gas clumps themselves are affected by the ram pressure, leading to structures with gaseous wakes, a kind of self-similarity.

In order to calculate comparable maps with observations we extracted mock X-ray and HI observations from our simulations. The X-ray surface brightness, X-ray temperature and

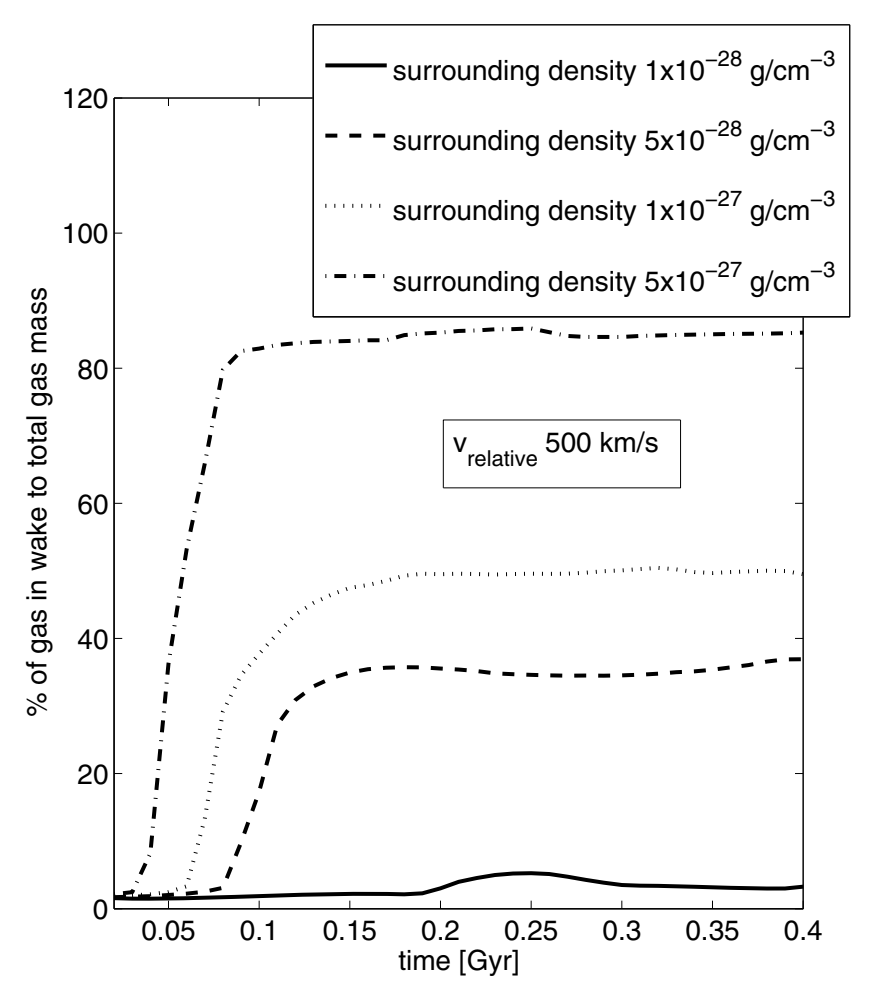

Fig. 14. Evolution of the gas mass in the wake to the total gas mass (distances larger than $10 \mathrm{kpc}$ above the disc).

$\mathrm{X}$-ray metal maps are produced as described in detail in Kapferer et al. (2007a,b). The initial metallicities of the galaxy were chosen to be constant for the gas in the disc and the surrounding gas, respectively. The values are 1 in solar abundances for the ISM 


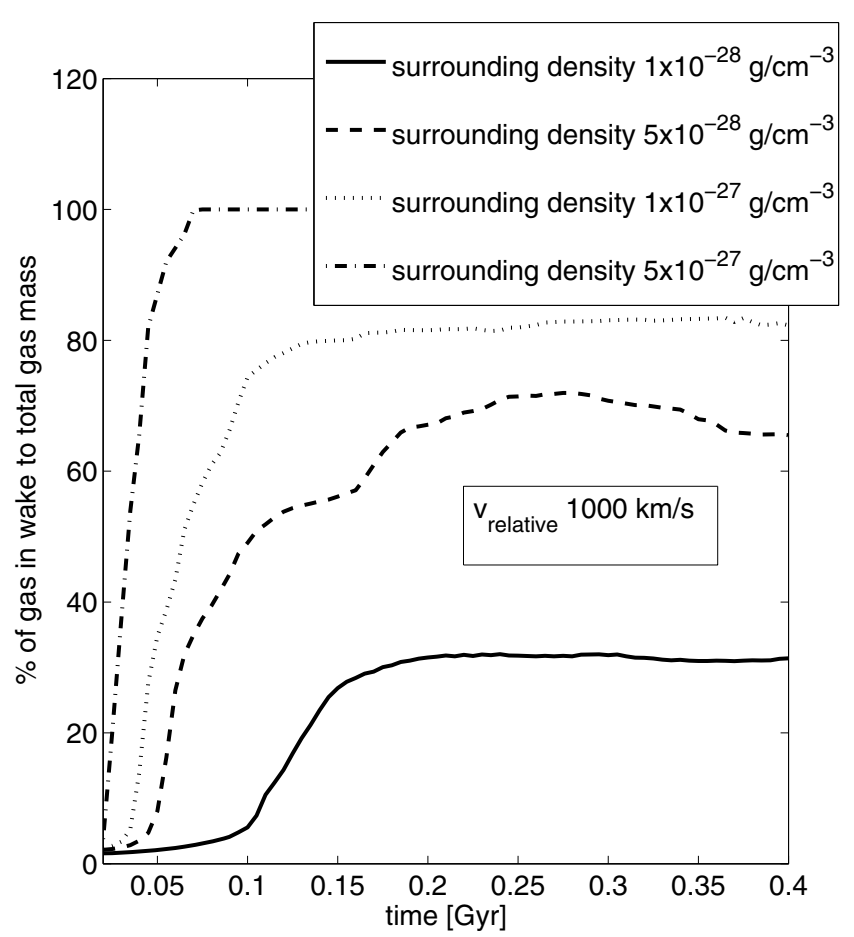

Fig. 15. Evolution of the gas mass in the wake to the total gas mass (distances larger than $10 \mathrm{kpc}$ above the disc).

and 0.3 in solar abundances for the hot, surrounding medium. Only gas with temperatures above $5 \times 10^{6} \mathrm{~K}$ is taken into account for the X-ray observations. For the HI mock observations we only take into account the cold $\left(T<2 \times 10^{5} \mathrm{~K}\right)$ gas. In addition we smooth the gas distribution with a Gaussian with $\sigma=4 \mathrm{kpc}$ to mimic a more realistic radio telescope beam size for galaxies in the distance of the Virgo cluster. The mock observations are shown in Figs. 22-24, respectively. In the case of simulation 1 (see Fig. 22) the resulting mock maps show an extended X-ray emitting wake with several hundred kpc extent. Roughly $20 \mathrm{kpc}$ and $150 \mathrm{kpc}$ behind the stellar disc a peak in the $\mathrm{X}$-ray maps is present. The latter is the region with the highest density of hot gas originating from the gaseous disc. In the X-ray temperature map a cool region of gas can be found at the same position, the X-ray metal map shows there the highest metallicities. The cold HI gas does not reach such large distances from the stellar disc. Nevertheless cool gas can be found in blobs at nearly $100 \mathrm{kpc}$ behind the disc. The effect of ram pressure can be seen in X-rays most dramatically, especially the X-ray metal map reveals many structures in the wake.

An increase of the ram pressure by one order of magnitude changes the HI observation dramatically, see Fig. 23. Nearly all cold gas is stripped, only a small fraction of cool gas is left in the centre of the disc. In addition to the cool gas the stellar surface brightness of newly formed stars is shown. The HI mock observations show a feature in opposite direction to the wake. The reason for the feature is cool gas, which was transported into the slipstream of the gaseous disc and as the disc protects this region from the external ram pressure the gas has fallen onto the disc, leading to features opposite to the direction of the ram pressure. These features are common throughout the simulations and survive typically some tens of Myr.

Similar to the case of simulation 1 an X-ray maximum in the surface brightness, temperature and metal map is seen behind the disc in the direction of the ram pressure. In addition a large region of X-ray emitting gas is observable at more than $300 \mathrm{kpc}$ behind the disc. This region is very prominent in the X-ray surface brightness and metal map. After 100 Myr of ram pressure the equilibrium state between the ram pressure on the disc and the restoring gravitational forces is nearly reached (see Fig. 15), therefore most of the material can be found at larger distances behind the disc, than in the ram pressure scenario of simulation 1. As the main ram-pressure stripping event has happened more than $400 \mathrm{Myr}$ before the mock observation, the gas has had already more time to mix with the surrounding gas, leading to less pronounced X-ray structures in the wake. In the right upper panel in Fig. 23 a stellar surface brightness up to a distance of $250 \mathrm{kpc}$ is shown. The asymmetry of the newly formed stars directly behind the disc originate from a recent stripping event of a gaseous spiral. Note, only newly formed stars within the last $500 \mathrm{Myr}$ are shown. The stellar components reach over the whole wake and end at the X-ray emitting region in a distance of $400 \mathrm{kpc}$. The strongest ram pressure of all simulations changes the global property of the X-ray emission to a great extent. The maximum at a distance of $400 \mathrm{kpc}$ marks the region at which the galaxy has passed when the gaseous disc was completely stripped, it is enclosed by cool gas. Many stars are formed at this location and as they fall back to the disc, they assemble to "highways" of stellar light.

The simulations with less strong ram-pressure show all a common feature, a long X-ray tail. Although not much of the gas cold gas is stripped off and the star formation takes place mainly in or very near the disc. A several tens of kpc long X-ray emitting hot gas tail develops. In Fig. 25 mock X-ray and $\mathrm{H} \alpha$ observations including isolines showing the distribution of the cold gas phase are presented for simulation 5 (surrounding gas density of $1 \times$ $10^{-28} \mathrm{~g} \mathrm{~cm}^{-3}$ and a relative velocity of $500 \mathrm{~km} \mathrm{~s}^{-1}$ ). The newly formed stars are mainly concentrated in the compressed residual disc. Like in the observations of Yoshida et al. (2002, 2004) some $\mathrm{H} \alpha$ emitting gas can be found several kpc behind the ram pressure affected disc in the forming gaseous wake.

\subsection{Comparison to other simulations of ram-pressure stripping}

Many of the results presented here are in good agreement with previously published results in other simulations of ram pressure stripping. In the literature (e.g. Roediger \& Brueggen 2007; Vollmer et al. 2001) the size of the unstripped disc is often compared to the analytic estimate of Gunn \& Gott (1972). This provides a convenient means of comparing simulations with different initial disc structures. We therefore checked the stripping radius for the simulations with a residual gaseous disc and found that the stripping radius lies within $10 \%$ of the predicted radius (Gunn \& Gott 1972; Domainko et al. 2006). As found in Vollmer et al. (2001) a considerable fraction of the stripped gas can fall back on to the disc. In our simulations this is due to the slipstream of the residual gaseous disc. As in the simulations of Schulz \& Struck (2001) the simulation presented here includes radiative cooling. The effect of the cooling is quite strong in the wake of the stripped gas, leading to lower temperatures and more dense regions, which will then form stars. The time scales for stripping are comparable to those found in Roediger et al. (2006) in the range of $100 \mathrm{Myr}$ or below and the overall sizes of the gaseous wakes comparable to those presented in Roediger et al. (2006). As the simulations presented in this work include feedback od star formation on the surrounding gas, the resulting properties of the gaseous wake are not as directly comparable to other simulations. A common difference is visible in the 

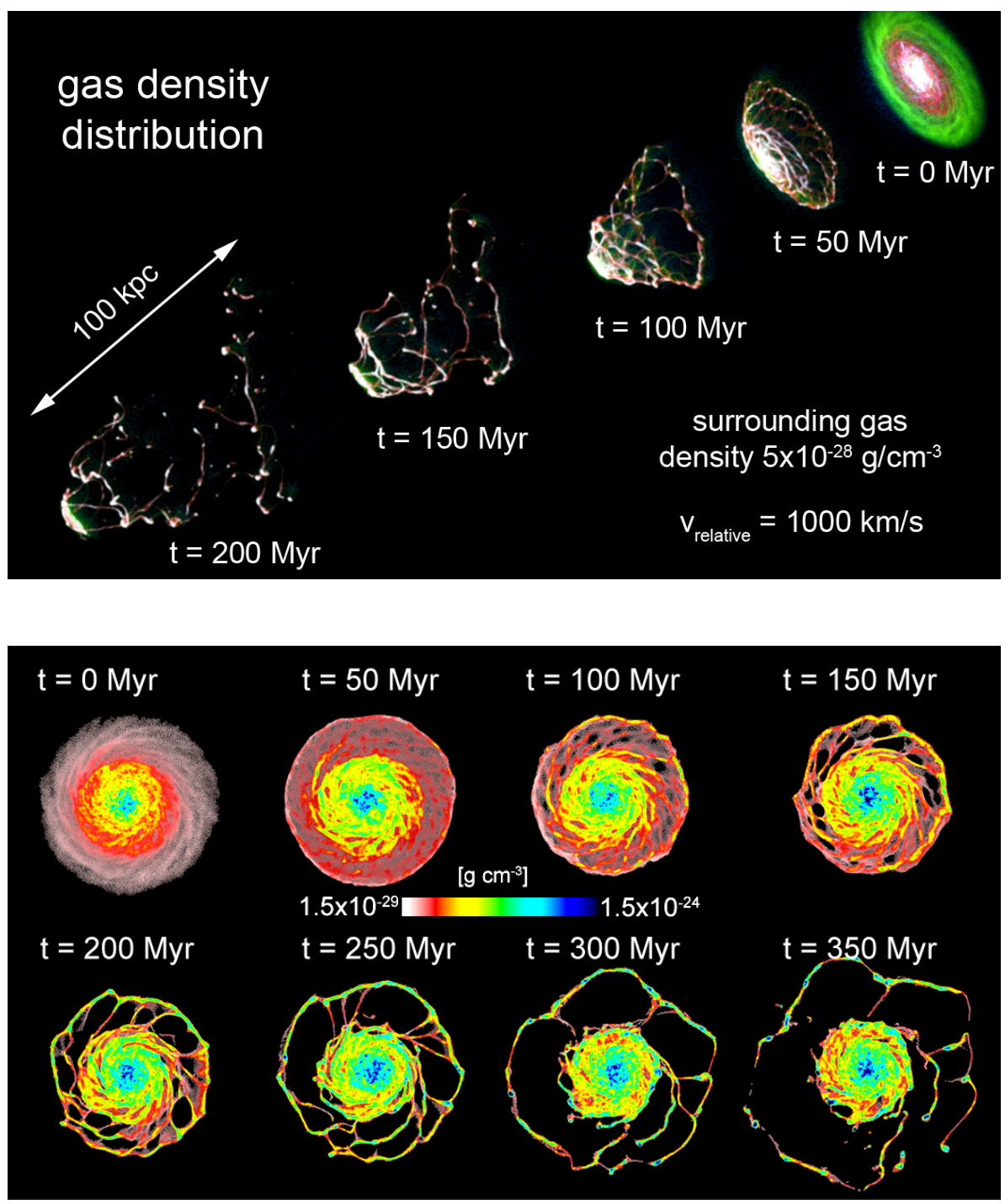

Fig. 16. The gas distribution for five different time steps, showing the evolution of the gas component over 200 Myr. The ram pressure and the relative velocity correspond to run 2 . The colour map indicates the density, i.e. white denser to green less dense regions.
Fig. 17. Gas density of the total gas in the disc for different timesteps for run 1 . The external ram pressure is $1 \times 10^{-12} \mathrm{dyn} / \mathrm{cm}^{2}$.

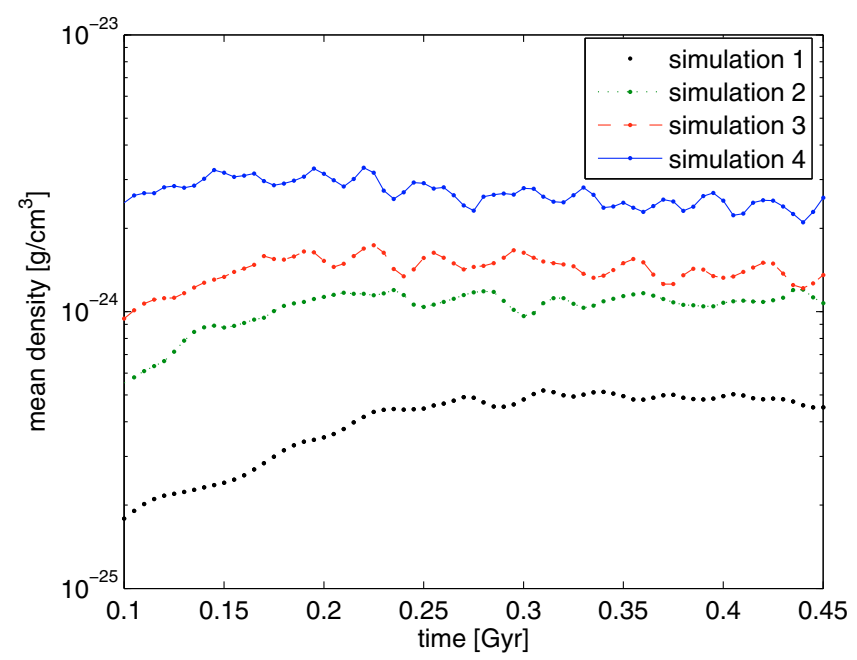

Fig. 18. Evolution of the mean local density of the cold $\left(T<10^{6} \mathrm{~K}\right)$ gas in the wake for simulations 1 to 4 (relative velocity $1000 \mathrm{~km} \mathrm{~s}^{-1}$ and surrounding gas density in the range from $1 \times 10^{-28}$ to $5 \times 10^{-27} \mathrm{~g} / \mathrm{cm}^{-3}$.

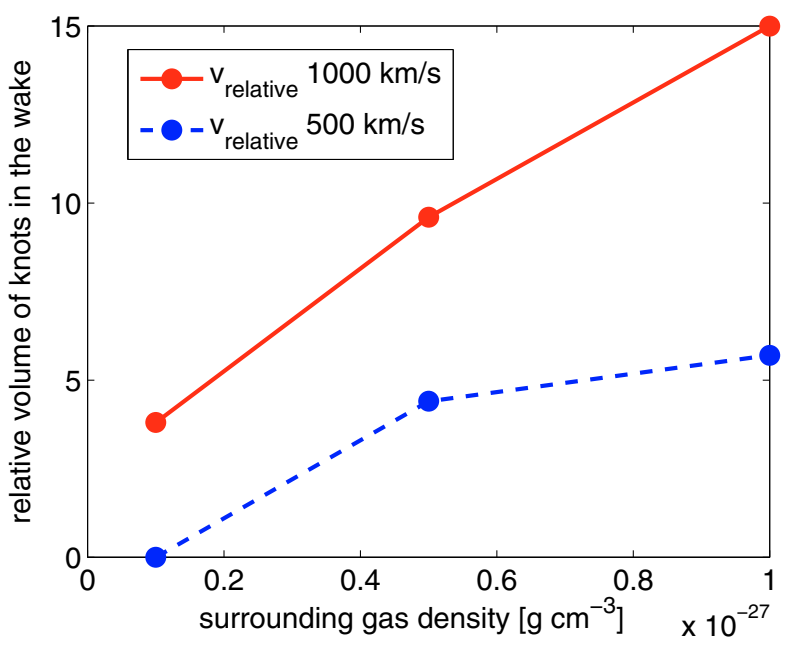

Fig. 19. Integrated volume of all isosurfaces with a fixed threshold of $1 \times 10^{5} M_{\odot} / \mathrm{kpc}^{3}$ calculated from the three dimensional gas density distribution in the wake as a function of surrounding gas density for the scenarios with $v_{\text {rel }}=500 \mathrm{~km} \mathrm{~s}^{-1}$ and $v_{\text {rel }}=1000 \mathrm{~km} \mathrm{~s}^{-1}$. 

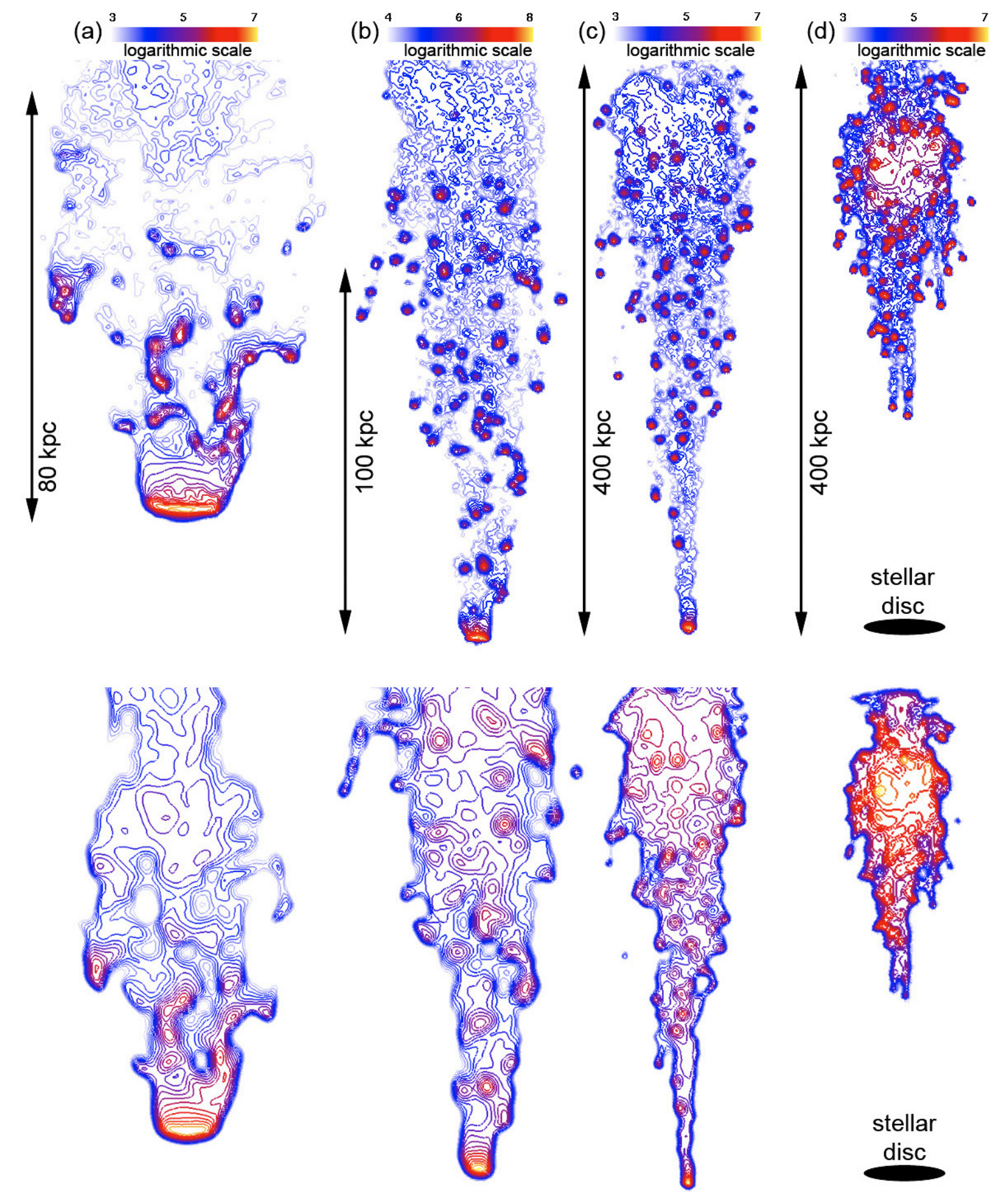

Fig. 20. Surface density isocontours of the total galaxies gas for different surrounding gas densities (a) $1 \times 10^{-28}$, b) $5 \times 10^{-28}$, c) $1 \times 10^{-27}$ and d) $5 \times 10^{-27} \mathrm{~g} \mathrm{c}^{-3}$ ) and a relative velocity of $1000 \mathrm{~km} \mathrm{~s}^{-1}$ after $500 \mathrm{Myr}$ of ram pressure. The upper panels show a resolution of $3 \mathrm{kpc}$ on a side for a cell, whereas the lower panel gives the same quantity smoothed with a Gaussian with $\sigma=4 \mathrm{kpc}$ to mimic a more realistic radio telescope beam size. In the case of the maximum ram pressure - d) - the gaseous disc is completely stripped and therefore the location of the old stellar disc is highlighted.

structure of the stripped wake, due to the high resolution in the presented simulations and the presence of spiral arms, the stripped gas has many small structures, which are stripped, evolve and survive in the wake over many hundreds of Myr. In Jáchym et al. (2007) for an example the stripped ISM does not show clumps of cool gas. In the eulerian grid simulations of Roediger et al. (2006) the wake does show cooler, dense regions, but the number is much less as in the simulations presented here.
A possible explanation is the lack of radiative cooling in the simulations by Jachym et al. (2007) and Reodiger et al. (2006).

\section{Comparison with observations}

Apart from the evidence for the effect of ram pressure on the gaseous component of galaxies seen in the radio wavelengths, recent observations in other wavebands have revealed the 
(a)
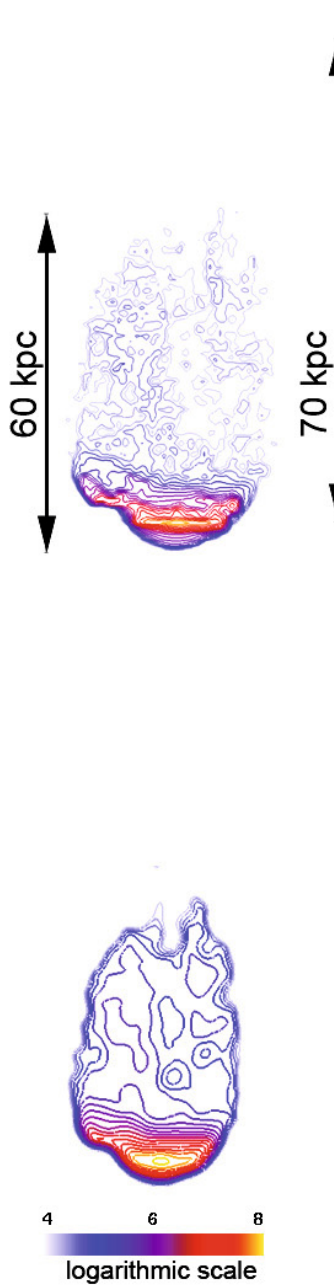

(b)
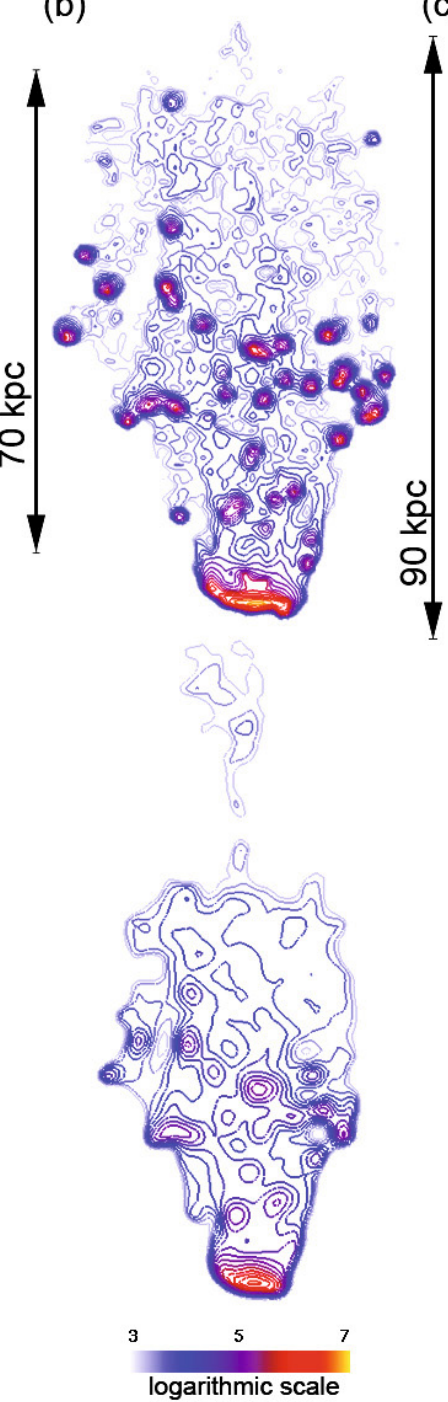

(c)
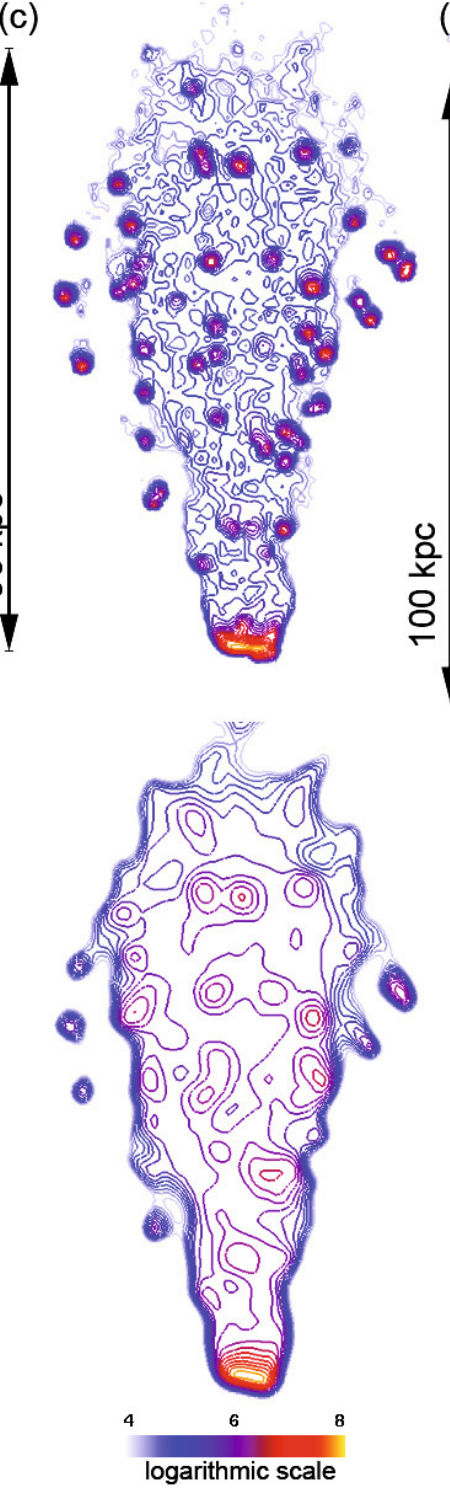

(d)
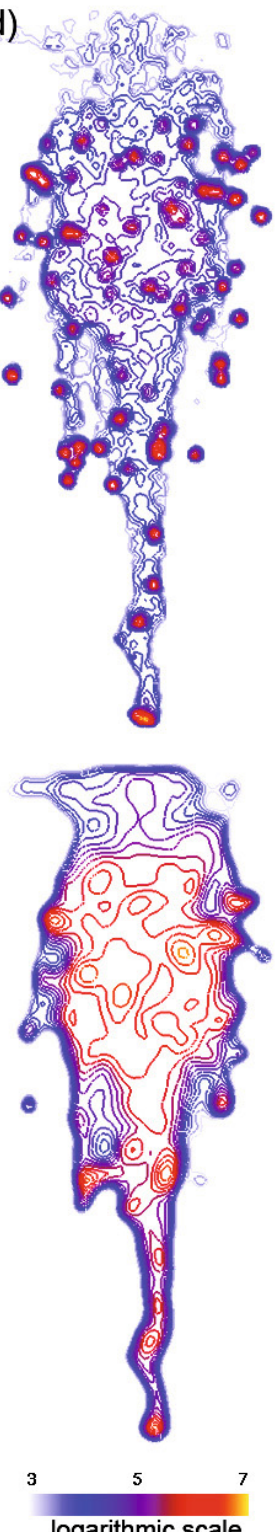

logarithmic scale

Fig. 21. Surface density isocontours of the total galaxies gas for different surrounding gas densities (a) $1 \times 10^{-28}$, b) $5 \times 10^{-28}$, c) $1 \times 10^{-27}$ and d) $5 \times 10^{-27} \mathrm{~g} \mathrm{c}^{-3}$ ) and a relative velocity of $500 \mathrm{~km} \mathrm{~s}^{-1}$ after $500 \mathrm{Myr}$ of ram pressure. The upper panels show a resolution of $3 \mathrm{kpc}$ on a side for a cell, whereas the lower panel gives the same quantity smoothed with a Gaussian with $\sigma=4 \mathrm{kpc}$ to mimic a more realistic radio telescope beam size.

influence of ram pressure on the stellar component. Yoshida et al. (2008) report on unusual complex narrow blue and $\mathrm{H} \alpha$ emitting filaments and clouds extending up to $80 \mathrm{kpc}$ south from an E+A galaxy RB199 in the Coma galaxy cluster. The galaxy seems to be a galaxy-galaxy merger. They conclude that the most plausible formation mechanism for these star forming regions is ram-pressure stripping.

Another example how ram pressure stripping influences the gaseous disc and the stripped material is presented in Yoshida et al. (2004, 2002). They investigated NGC 4388, a Seyfert 2 Virgo cluster galaxy, with deep optical spectroscopy. Many $\mathrm{H} \alpha$ regions in several tens of kpc in the stripped wake were found. One explanation for the off-disc $\mathrm{H} \alpha$ emission is the Seyfert nucleus, acting as a ionization source. Another mechanism is shocks introduced by the ram pressure, which ionizes the cloud complexes. Some of the gas clouds found have masses in the range of $10^{5}$ to $10^{6} M_{\odot}$ and sizes up to several hundred pc.
The excitation mechanism in the outer region of the stripped cloud complexes $(r>12 \mathrm{kpc})$ is yet unclear. In the simulations presented here, several comparable star forming knots were found in the stripped wake. As in these gas complexes star formation is present, one explanation for the observed $\mathrm{H} \alpha$ emission in the gas complexes far from the Seyfert nucleus in NGC 4388 is ongoing star formation, comparable to the mock observations of simulation 5 presented in Fig. 25.

Another striking observation is presented by Sun et al. (2007). They find a $40 \mathrm{kpc} \mathrm{H} \alpha$ tail and at least 29 emission-line objects downstream a star-forming galaxy in the the galaxy cluster A3627. The galaxy has in addition to the $\mathrm{H} \alpha$ tail an X-ray tail with a length of $70 \mathrm{kpc}$, very similar to the stripping situation in shown in Fig. 23. Both can be found in our simulations as well.

The longest X-ray tail formed from stripped gas originating from a galaxies observed so far is located in the Virgo galaxy cluster (Randall et al. 2008), a Chandra observation of M 86 in 

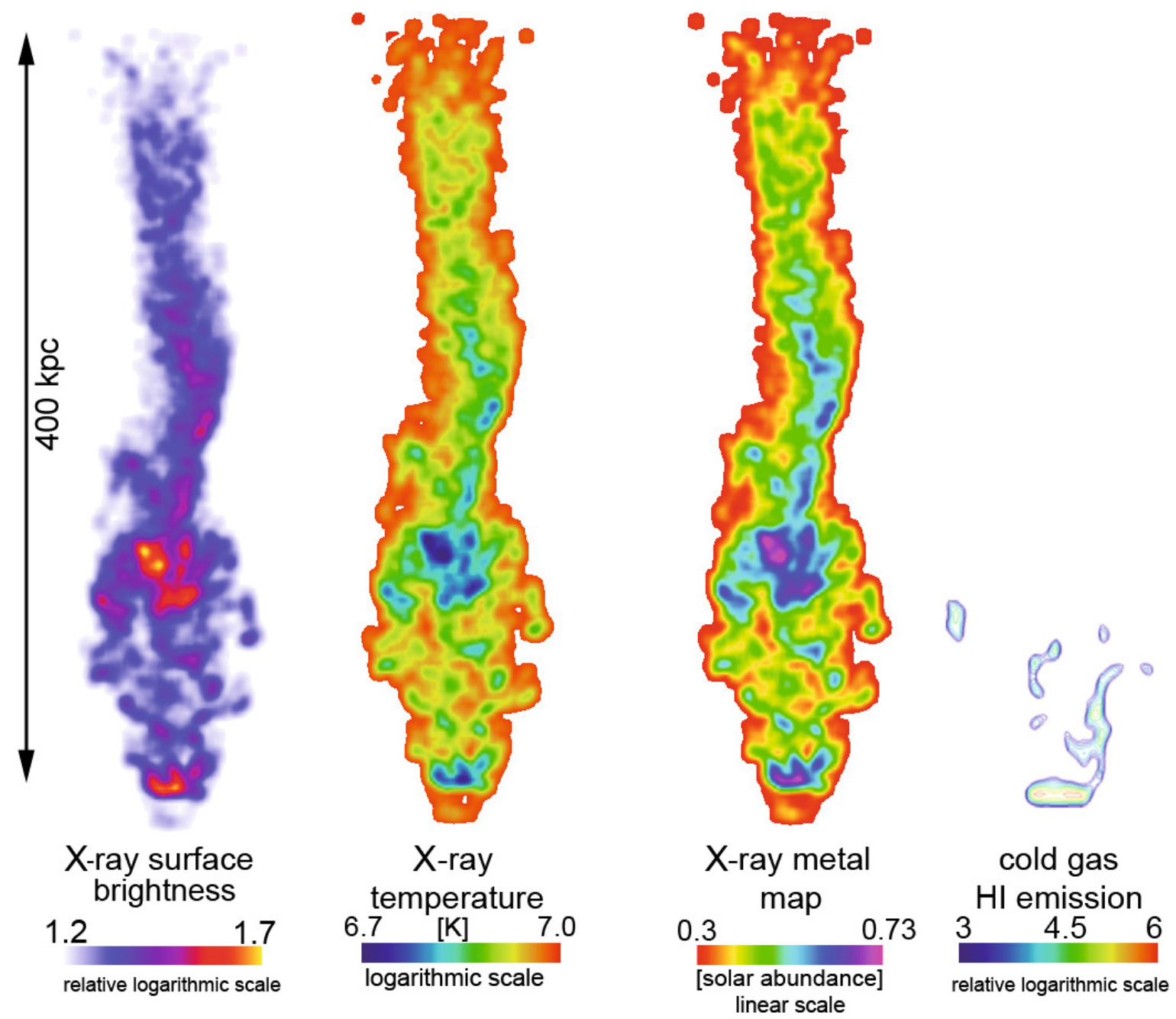

Fig. 22. Mock X-ray and $\mathrm{HI}$ observations for simulation 1, i.e. $1000 \mathrm{~km} \mathrm{~s}^{-1}$ relative velocity and a surrounding gas density of $1 \times 10^{-28} \mathrm{~g} \mathrm{~cm}{ }^{-3}$. The ram pressure has acted for $500 \mathrm{Myr}$ on the model galaxy.

Virgo and the surrounding field. The X-ray tail is $150 \mathrm{kpc}$ in projection and a simple estimate of the true trajectory yields an $\mathrm{X}$-ray tail with a length of $380 \mathrm{kpc}$. In addition they find an X-ray plume behind the X-ray emission of M 86, very similar to our finding that the first X-ray maximum can be found behind the ram-pressure affected disc, as a result of the first compressional heating of the stripped gas.

Recently Kim et al. (2008) presented Chandra and XMM-Newton observations of a prominent X-ray tail of the galaxy NGC 7619. They found in the tail a significantly higher metallicity than in the surrounding regions, and conclude that this indicates, that the gas originates from the galaxy. In addition they find discontinuities in the X-ray surface brightness map in the opposite direction, which suggest a supersonic relative velocity of the galaxy with respect to the surrounding gas.

The origin of intergalactic HI complexes is still under debate. A good example is VIRGOHI21 (Davies et al. 2004; Minchin et al. 2005). This very massive (from dynamical estimates $\approx 10^{11} M_{\odot}$ ) dark galaxy could either be the result of a high velocity encounter (Duc \& Bournaud 2008) or simply gas trapped in a dark matter halo, not yet forming stars. Taking the results of this simulation into account another possibility arise, VIRGOHI21 could be the result of a strong stripping event. Like in Fig. 24 nearly to whole gaseous disc could be stripped by a single stripping event. The gas complex, as not bound to a dark matter halo, would not form stars on time scales comparable to the same amount of gas trapped in a dark matter halo.

\section{Discussion and conclusions}

We present numerical studies of the influence of ram pressure on disc galaxies and their stellar and gaseous components. We performed 12 different high resolution SPH simulations with the same mass resolution for the gas in the disc and the surrounding gas. The simulations include radiative cooling and a recipe for star formation (Springel \& Hernquist 2003). We varied the density of the surrounding gas from $1 \times 10^{-28}$ to $5 \times 10^{-27} \mathrm{~g} / \mathrm{cm}^{3}$ and the relative velocity of the galaxy with respect to the surrounding gas from $100 \mathrm{~km} \mathrm{~s}^{-1}$ to $1000 \mathrm{~km} \mathrm{~s}^{-1}$. The chosen combinations probe the ram pressure range from $1 \times 10^{-12}$ to $5 \times 10^{-13} \mathrm{dyn}^{\mathrm{cm}}{ }^{2}$. The surrounding gas temperature was chosen to be $1 \times 10^{7} \mathrm{~K}$, this leads to ICM conditions similar to many regions in the Virgo galaxy cluster (Schindler et al. 1999). The study here can be seen as an extension of the studies presented by Kronberger et al. (2008) and Kapferer et al. (2008), including mock observations in different wavelength, like in the $\mathrm{HI}, \mathrm{H} \alpha$ and X-ray range. The results of this work can be summarised as follows:

- The star-formation rate for simulations with ram pressure can be more then 10 times higher then for the same galaxy 

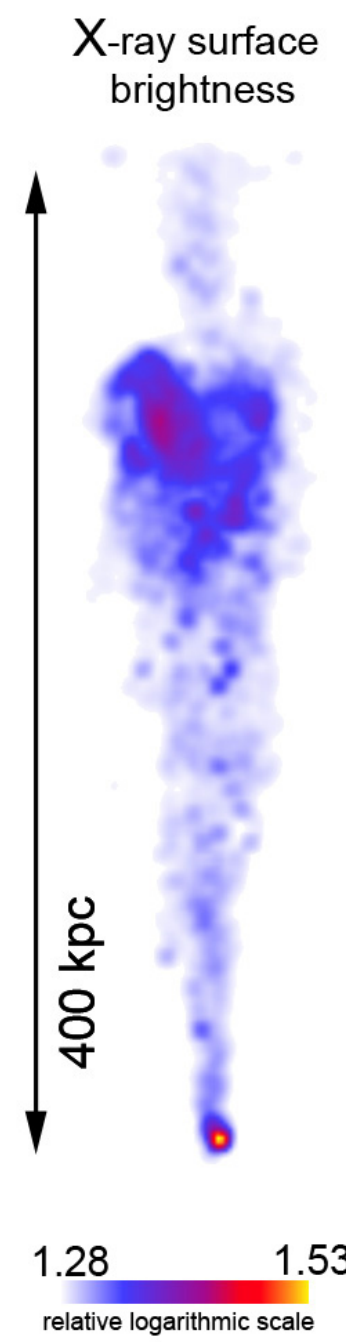
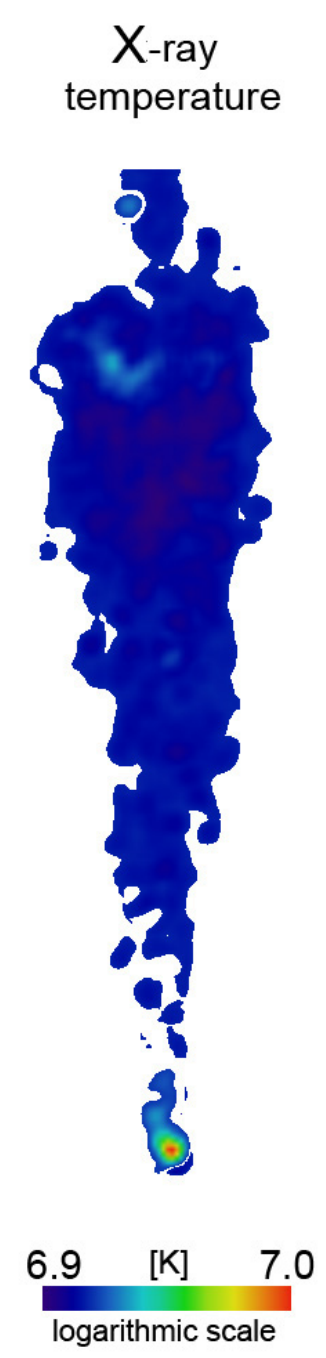
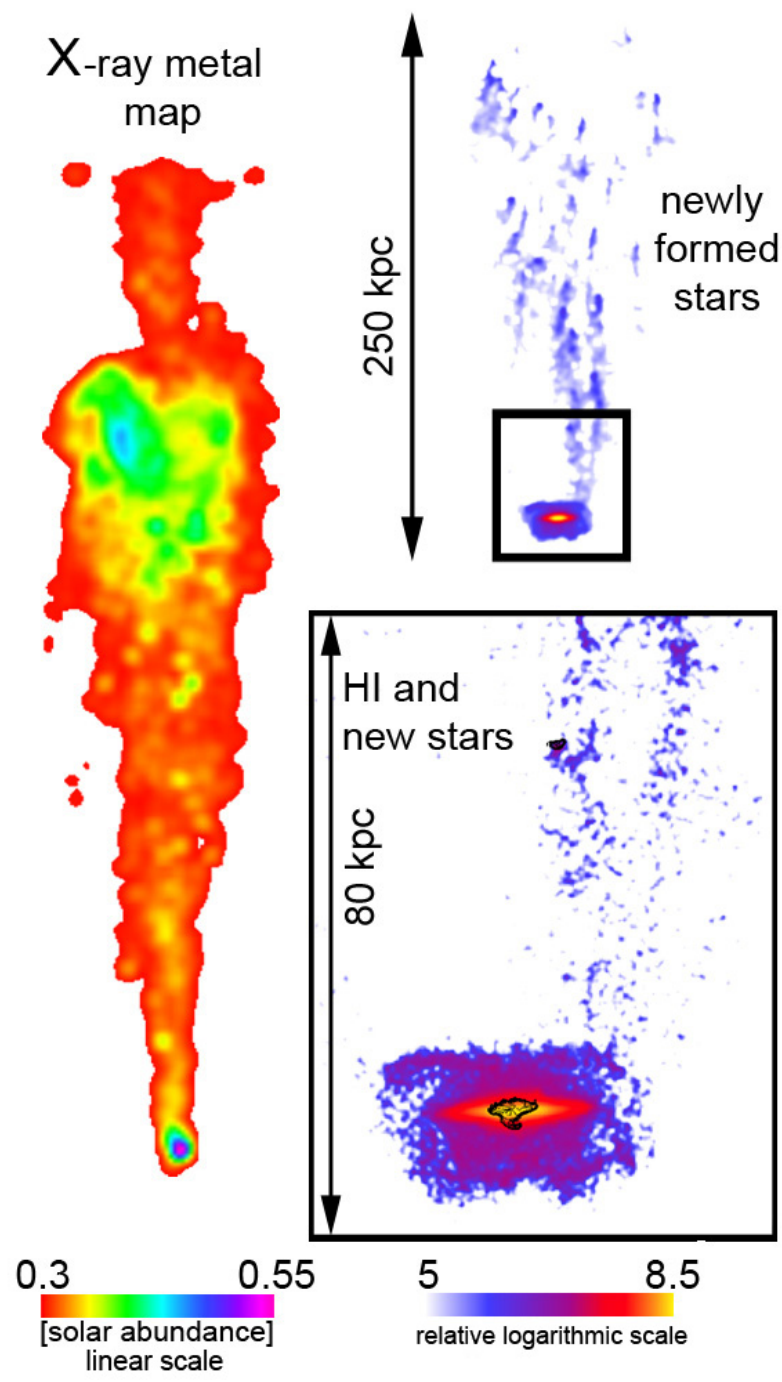

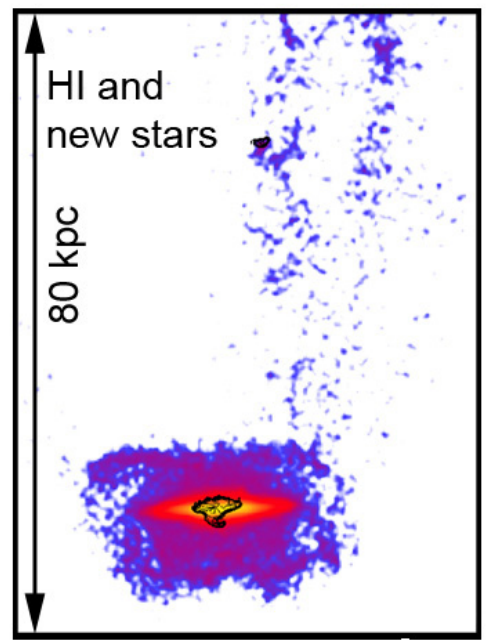

0.55

8.5 relative logarithmic scale

Fig. 23. Mock X-ray and HI observations for simulation 3, i.e. $1000 \mathrm{~km} \mathrm{~s}^{-1}$ relative velocity and a surrounding gas density of $1 \times 10^{-27} \mathrm{~g} \mathrm{~cm}{ }^{-3}$. The ram pressure has acted $500 \mathrm{Myr}$ on the model galaxy.

evolving in vacuum. The star-formation rate depends more strongly on the surrounding gas density than on the relative velocity. This can be explained by the location of the newly formed stars. The higher the ram pressure, the more gas is stripped from the galaxy, leading to less star formation in the disc. On the other hand more star-forming gaseousknots are present in the gaseous wake, which form many new stars. The higher the external pressure on this knots, the more stars are formed. For surrounding gas densities above $5 \times$ $10^{-27} \mathrm{~g} / \mathrm{cm}^{3}$ the star formation is the highest for the lowest relative velocity. For lower gas densities this trend reverses.

- The distribution of newly formed stars depends strongly on the ram pressure on the galaxy. More than $95 \%$ of all the newly formed stars in the simulation with the maximum ram pressure are located in the stripped wake of the galaxy (simulation 4). The stars are formed from the stripped gas originating from the galaxy at larger distances, up to $400 \mathrm{kpc}$ behind the disc after $500 \mathrm{Myr}$. If the relative velocity drops below $500 \mathrm{~km} \mathrm{~s}^{-1}$ and the surrounding gas density is below $\rho \leq 1 \times 10^{-28} \mathrm{~g} / \mathrm{cm}^{3}$ all the new stars are formed in the disc.

- The surface density of stars in the wake is typically 3 to 4 mag lower than in the central regions of the stellar disc. The locations of newly formed stars can be found hundreds of kpc behind the disc, depending on the strength and duration of the ram pressure. Due to the fact that newly formed stars are not feeling the ram pressure anymore, their trajectories are only influenced by the underlying gravitational potential. They fall back to the centre of the mass distribution, which is the stellar disc. As the stars are treated as a collisionless fluid they are falling through the stellar disc and appear in the opposite direction of the wake. This leads to stellar bulges around the old stellar disc, they are more pronounced in the opposite direction of the ram pressure.

- A young stellar population at a given time (formed within the last $50 \mathrm{Myr}$ ), which could be called OB-associations, is detectable throughout the whole wake. In the initial ram pressure event, the first 50 to $200 \mathrm{Myr}$ depending on the strength of the ram pressure, they are very prominent in the whole stellar component present in the wake. After the initial stripping event, the OB-associations are found at the locations of the largest gaseous densities in the wake, the regions were the new stars are formed. Their surface density is locally of the same order of magnitude as in the stellar disc, if the ram pressure does not strip the whole gas of the disc.

- If the model galaxy is affected by strong external ram pressure the positions of star formation are shifted from the disc to the wake. In observations this would lead to a classification of the disc galaxy as a passively evolving system, 


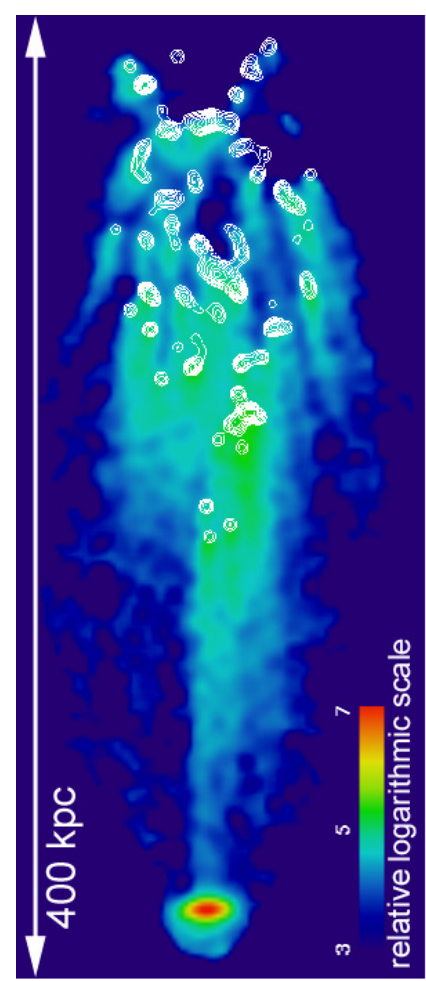

$\mathrm{HI}$ and new stars
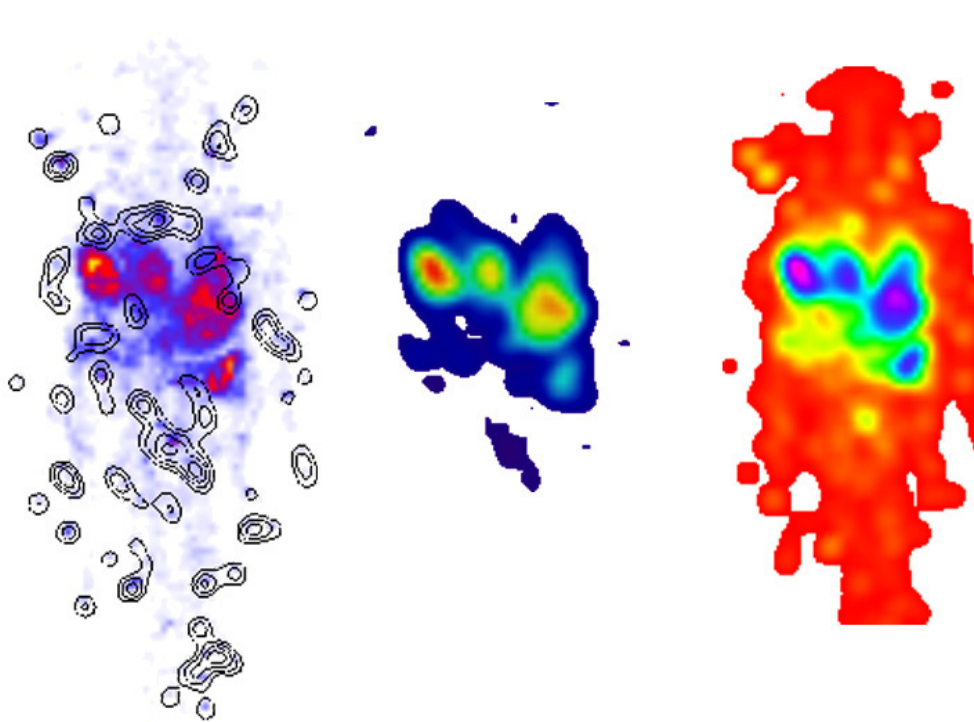

()

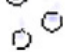

1.7

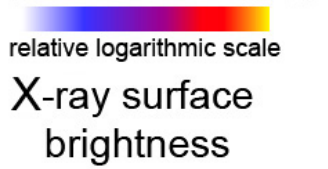

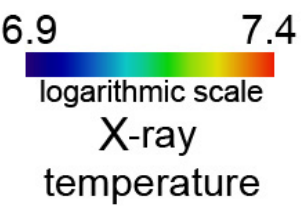
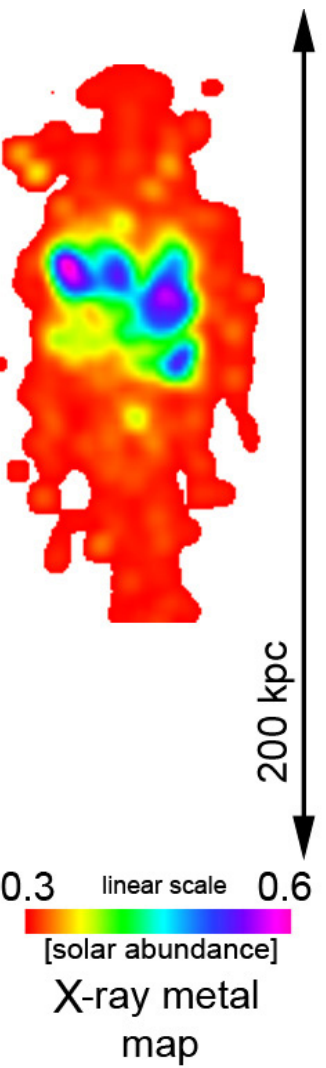

Fig. 24. Mock X-ray and $\mathrm{HI}$ observations for simulation 4 , i.e. $1000 \mathrm{~km} \mathrm{~s}^{-1}$ relative velocity and a surrounding gas density of $5 \times 10^{-27} \mathrm{~g} \mathrm{~cm}^{-3}$. The ram pressure has acted 500 Myr on the model galaxy, it is the simulation in which the gaseous disc as completely stripped from the galaxy. $\mathrm{X}$-ray temperature map in $\mathrm{K}$.

although the overall star formation rate (disc and wake) is strongly enhanced by the ram pressure. The time scale can be very short, in our strongest ram-pressure stripping simulation it is less than $80 \mathrm{Myr}$.

- The influence of ram pressure on the gaseous component depends strongly on the strength of the ram pressure. In the case of simulation $4\left(v_{\text {rel }}=1000 \mathrm{~km} \mathrm{~s}^{-1}\right.$ and a surrounding gas density of $5 \times 10^{-27} \mathrm{~g} / \mathrm{cm}^{3}$ ) the gaseous disc is stripped completely after 75 Myr of ram pressure. By reducing the relative velocity by a factor of $2\left(500 \mathrm{~km} \mathrm{~s}^{-1}\right)$ the disc is stripped off more then $80 \%$ of the initial gas mass in the disc. As the gas in the galaxy has an internal velocity pattern from the rotational velocity field of the galaxy, the stripped gas has complex structures in the stripped wake. As a result, the stripped wake forms filaments and gas concentrations. The self-gravitating gas concentrations survive throughout the whole simulation time (more than $750 \mathrm{Myr}$ ).

- The number of dense gaseous knots, which act as starforming cocoons increases with the strength of the ram pressure. In the simulation with the highest ram pressure $\left(v_{\text {rel }}=\right.$ $\left.1000 \mathrm{~km} \mathrm{~s}^{-1} \rho_{\text {sur }}=1 \times 10^{-27} \mathrm{~g} / \mathrm{cm}^{3}\right)$ the number of gaseous knots with a surface density above $1 \times 10^{5} M_{\odot} / \mathrm{kpc}^{3}$ is three times higher than in the case with $\rho_{\text {sur }}=1 \times 10^{-28} \mathrm{~g} / \mathrm{cm}^{3}$.

- The mock X-ray observations of the simulations reveal X-ray tails with lengths of several hundred $\mathrm{kpc}$, depending on the strength of the ram pressure. Complex structures can be found in the X-ray surface brightness, temperature and metal maps. One common feature is a bright spot in all X-ray maps behind the stellar disc at distances of a few tens of kpc caused by compressional heating of the stripped gas. The location of the initial stripping event can be seen nicely as the second bright feature in the X-ray maps. The stronger the ram pressure, the more pronounced the feature. In the simulations with $1000 \mathrm{~km} \mathrm{~s}^{-1}$ and a surrounding gas density of $1 \times 10^{-28} \mathrm{~g} / \mathrm{cm}^{3}$ the X-ray tail is longer than in the case with ten times higher surrounding gas density. This leads to the conclusion that a strong ram-pressure event, stripping off the whole gaseous disc within the initial stripping event, produces metal structures in the X-ray maps which are spatially more concentrated than in events with less ram pressure.

- The cold stripped gas is not as widely spread in the wake as the X-ray emitting hot gas. It can be mainly found near the location of the first stripping event and near the stripped disc. Even features in the opposite direction of the wake can be found. The reaccretion of cold gas in the wake, which falls back onto the disc as it enters the slipstream of the remaining gas in the disc is the explanation for this. If the gaseous disc is completely stripped, cold gas is spread over more then $150 \mathrm{kpc}$ around the maxima in the X-ray surface brightness, temperature and metal map.

Ram pressure stripping changes the stellar- and the gaseous distribution in galaxies drastically. Therefore the investigation of this external process is crucial for understanding galaxy evolution in galaxy clusters or groups. As the timescales occurring in ram-pressure stripping events are fast compared to internal processes in the disc, the transition from an actively to a passively evolving system can occur on very short timescales. Due to observational limits the conclusion that a system is passively evolving can be misleading as the total star formation is typically enhanced in a ram-pressure event, but the location of star formation 

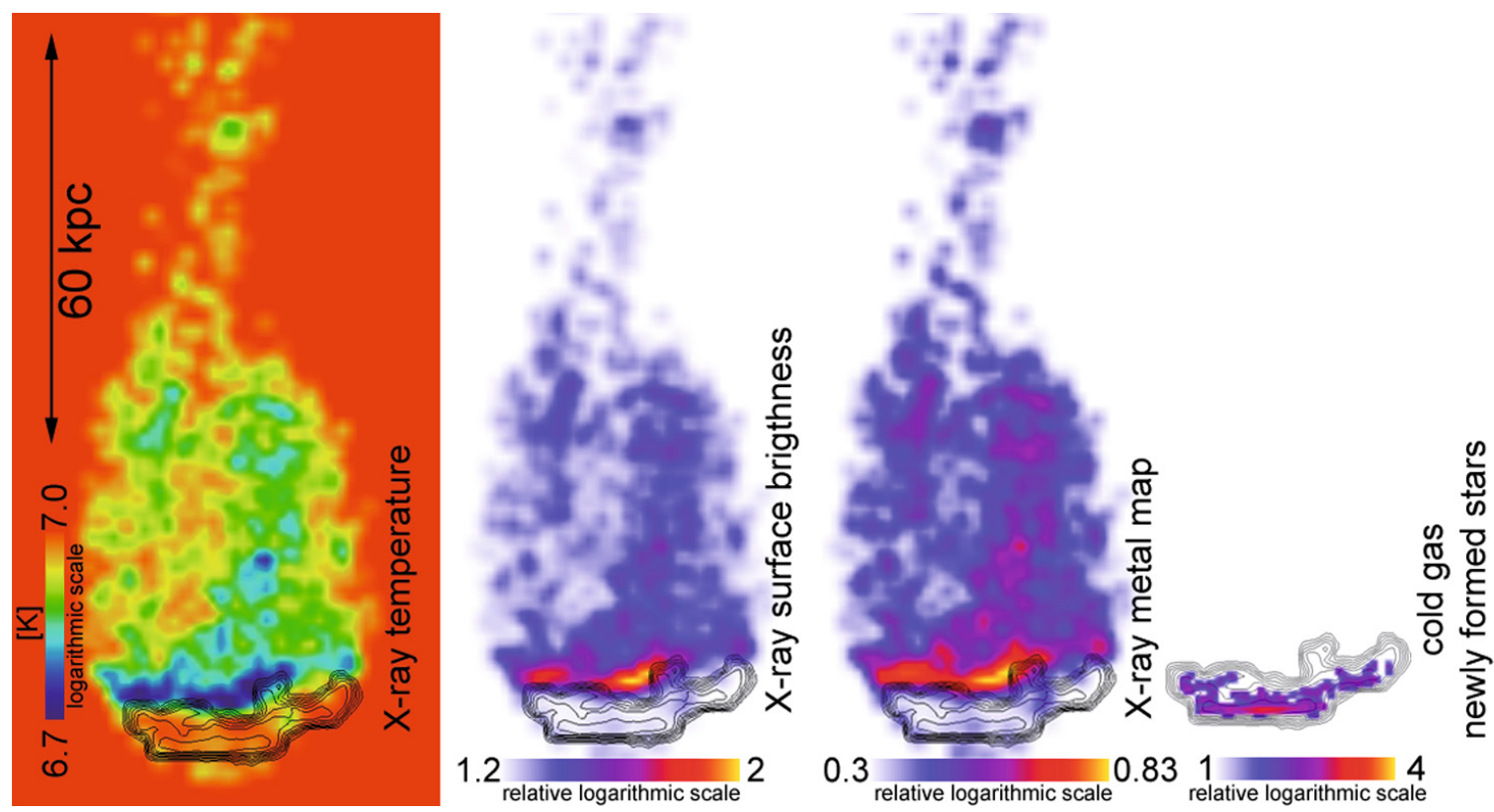

Fig. 25. Mock X-ray and HI observations and newly formed stars for simulation 5, i.e. $500 \mathrm{~km} \mathrm{~s}^{-1}$ relative velocity and a surrounding gas density of $1 \times 10^{-28} \mathrm{~g} \mathrm{~cm}^{-3}$. The ram pressure has acted for $500 \mathrm{Myr}$ on the model galaxy, in this, the simulation with the weakest stripping.

is moved from the disc into the stripped wake. Another implication of the presented results affects observations of metal enrichment processes in the ICM. The element abundances originating from galactic winds and ram pressure stripping are maybe very similar. The presence of ram-pressure induced intra-cluster star formation makes it very difficult to distinguish between gas removed by galactic winds or gas removed by ram pressure. Further investigations are needed to disentangle the influence of the different processes on the chemical composition of the intracluster medium.

How other effects, like magnetic fields or different heating and cooling processes, due to a complex mixture of different gas phases, would change the presented numbers needs further investigations as well. Recent observational findings and the presented simulations here reveal a luminous universe outside the bright spots, the galaxies.

Acknowledgements. The authors would like to thank the referee Curtis Struck for his constructive suggestions, which increased the quality of the paper. The authors thank Volker Springel for providing them with GADGET2. The authors are grateful to Marco Barden for many useful discussions and for Sabine Kreidl and Gerhard Niederwieser for the perfect support at the Computational Centre at the University of Innsbruck. The authors acknowledge the Austrian Science Foundation (FWF) through grants P18523-N16 and P19300-N16. The authors further acknowledge the UniInfrastrukturprogramm des BMWF Forschungsprojekt Konsortium Hochleistungsrechnen.

\section{References}

Abadi, M. G., Moore, B., \& Bower, R. G. 1999, MNRAS, 308, 947 Arnaboldi, M., Freeman, K. C., Okamura, S., et al. 2003, AJ, 125, 514 Balogh, M., Bower, R. G., Smail, I., et al. 2002, MNRAS, 337, 256 Breitschwerdt, D., McKenzie, J. F., \& Völk, H. J. 1991, A\&A, 245, 79 Butcher, H., \& Oemler, A., Jr. 1978, ApJ, 219, 18

Chemin, L., Balkowski, C., Cayatte, V., et al. 2006, MNRAS, 366, 812 Davies, J., Minchin, R., Sabatini, S., et al. 2004, MNRAS, 349, 922 Domainko, W., Mair, M., Kapferer, W., et al. 2006, A\&A, 452, 795 Dressler, A. 1987, Nearly Normal Galaxies: From the Planck Time to the Present, ed. S. M. Faber (New York: Springer-Verlag), 163.276 Dressler, A., Smail, I., Poggianti, B. M., et al. 1999, ApJS, 122, 51 Duc, P.-A., \& Bournaud, F. 2008, ApJ, 673, 787

Feldmeier, J. J., Ciardullo, R., \& Jacoby, G. H. 1998, ApJ, 503, 109 Ganda, K., Falcón-Barroso, J., Peletier, R. F., et al. 2006, MNRAS, 367, 46
Garrido, O., Marcelin, M., Amram, P., \& Boulesteix, J. 2002, A\&A, 387, 821 Gerken, B., Ziegler, B., Balogh, M., et al. 2004, A\&A, 421, 59 Gingold, R. A., \& Monaghan, J. J. 1977, MNRAS, 181, 375

Gunn, J. E., \& Gott, J. R. I. 1972, ApJ, 176, 1

Jáchym, P., Palouš, J., Köppen, J., \& Combes, F. 2007, A\&A, 472, 5

Kapferer, W., Kronberger, T., Schindler, S., Böhm, A., \& Ziegler, B. L. 2006, A\&A, 446, 847

Kapferer, W., Kronberger, T., Weratschnig, J., et al. 2007a, A\&A, 466, 813

Kapferer, W., Kronberger, T., Weratschnig, J., \& Schindler, S. 2007b, A\&A, 472, 757

Kapferer, W., Kronberger, T., Ferrari, C., Riser, T., \& Schindler, S. 2008, MNRAS, 389, 1405

Kim, D.-W., Kim, E., Fabbiano, G., \& Trinchieri, G. 2008, ApJ, 688, 931 Kronberger, T., Kapferer, W., Schindler, S., et al. 2006, A\&A, 458, 69 Kronberger, T., Kapferer, W., Schindler, S., \& Ziegler, B. L. 2007, A\&A, 473, 761

Kronberger, T., Kapferer, W., Ferrari, C., Unterguggenberger, S., \& Schindler, S. 2008, A\&A, 481, 337

Larson, R. B., Tinsley, B. M., \& Caldwell, C. N. 1980, ApJ, 237, 692 Lucy, L. B. 1977, AJ, 82, 1013

Maurogordato, S., Cappi, A., Ferrari, C., et al. 2008, A\&A, 481, 593 Mihos, C., Harding, P., Feldmeier, J., \& Morrison, H. 2005, BAAS, 37, 1449

Minchin, R., Davies, J., Disney, M., et al. 2005, ApJ, 622, L21

Moore, B., Lake, G., \& Katz, N. 1998, ApJ, 495, 139

Mori, M., \& Burkert, A. 2000, ApJ, 538, 559

Poggianti, B. M., et al. 2006, ApJ, 642, 188

Quilis, V., Moore, B., \& Bower, R. 2000, Science, 288, 1617

Randall, S., Nulsen, P., Forman, W. R., et al. 2008, ApJ, 688, 208

Roediger, E., \& Hensler, G. 2005, A\&A, 433, 875

Roediger, E., \& Brüggen, M. 2006, MNRAS, 369, 567

Roediger, E., \& Brüggen, M. 2007, MNRAS, 380, 1399

Roediger, E., \& Brüggen, M. 2008, MNRAS, 388, 465

Rubin, V. C., Waterman, A. H., \& Kenney, J. D. P. 1999, AJ, 118, 236

Russell, H. R., Sanders, J. S., \& Fabian, A. C. 2008, MNRAS, 390, 1207

Schindler, S., Binggeli, B., \& Böhringer, H. 1999, A\&A, 343, 420

Schindler, S., Kapferer, W., Domainko, W., et al. 2005, A\&A, 435, L25

Schulz, S., \& Struck, C. 2001, MNRAS, 328, 185

Springel, V. 2005, MNRAS, 364, 1105

Springel, V., \& Hernquist, L. 2003, MNRAS, 339, 289

Sun, M., Donahue, M., \& Voit, G. M. 2007, ApJ, 671, 190

Theuns, T., \& Warren, S. J. 1997, MNRAS, 284, L11

Toniazzo, T., \& Schindler, S. 2001, MNRAS, 325, 509

Verdugo, M., Ziegler, B. L., \& Gerken, B. 2008, A\&A, 486, 9

Vollmer, B., Cayatte, V., Balkowski, C., \& Duschl, W. J. 2001, ApJ, 561, 708

Yoshida, M., Yagi, M., Okamura, S., et al. 2002, ApJ, 567, 118

Yoshida, M., Ohyama, Y., Iye, M., et al. 2004, AJ, 127, 3653

Yoshida, M., Yagi, M., Komiyama, Y., et al. 2008, ApJ, 688, 918 